\title{
A mixed-mode phase field fracture model in anisotropic rocks with consistent kinematics
}

\author{
Eric C. Bryant, WaiChing Sun* \\ Department of Civil Engineering and Engineering Mechanics, Columbia University, 614 SW Mudd, Mail Code: 4709, New York, NY 10027, \\ United States
}

Received 17 May 2018; received in revised form 1 August 2018; accepted 6 August 2018

Available online 18 August 2018

\begin{abstract}
Under a pure tensile loading, cracks in brittle, isotropic, and homogeneous materials often propagate such that pure mode I kinematics are maintained at the crack tip. However, experiments performed on geo-materials, such as sedimentary rock, shale, mudstone, concrete and gypsum, often lead to the conclusion that the mode I and mode II critical fracture energies/surface energy release rates are distinctive. This distinction has great influence on the formation and propagation of wing cracks and secondary cracks from pre-existing flaws under a combination of shear and tensile or shear and compressive loadings. To capture the mixed-mode fracture propagation, a mixed-mode I/II fracture model that employs multiple critical energy release rates based on Shen and Stephansson, IJRMMS, 1993 is reformulated in a regularized phase field fracture framework. We obtain the mixedmode driving force of the damage phase field by balancing the microforce. Meanwhile, the crack propagation direction and the corresponding kinematics modes are determined via a local fracture dissipation maximization problem. Several numerical examples that demonstrate mode II and mixed-mode crack propagation in brittle materials are presented. Possible extensions of the model capturing degradation related to shear/compressive damage, as commonly observed in sub-surface applications and triaxial compression tests, are also discussed.
\end{abstract}

(C) 2018 Elsevier B.V. All rights reserved.

Keywords: Mixed-mode fracture; Secondary crack; Phase field fracture

\section{Introduction}

Brittle fracture process in geological materials can be explained by Griffith theory [1], which provides the linkages among stress concentration caused by sharp-tipped flaws, the energy flux, and the conditions for propagation of various types of flaws. The popularity of fracture mechanics' application to geomaterials is largely due to its simplicity, as well as capacity to predict the growth and spreading of the flaws $[2,3]$.

In the brittle regime where confining pressure, temperature, and loading rate are sufficient low, Griffith theory provides convenient tools to analyze the onset and early propagation of mode I cracks in a homogeneous, isotropic, and

\footnotetext{
* Corresponding author.

E-mail address: wsun@ columbia.edu (W.C. Sun).
} 


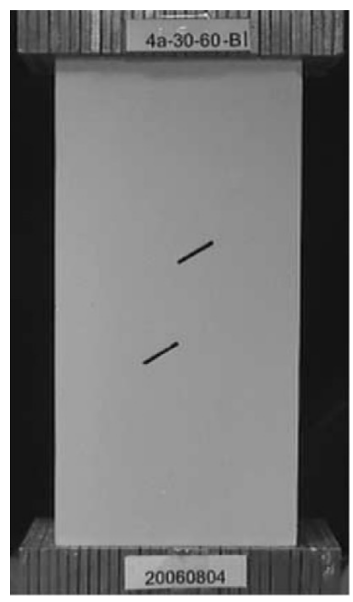

(a)

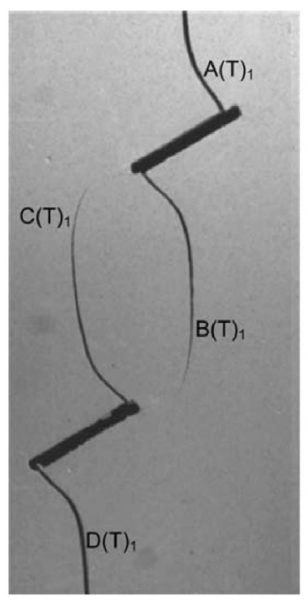

(b)

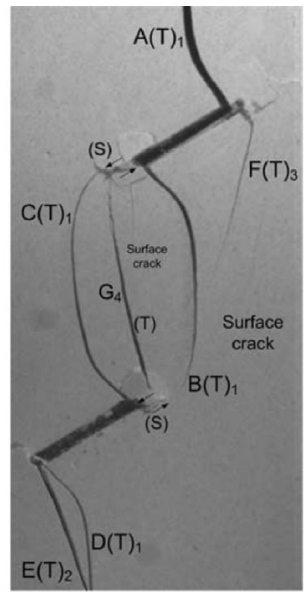

(c)

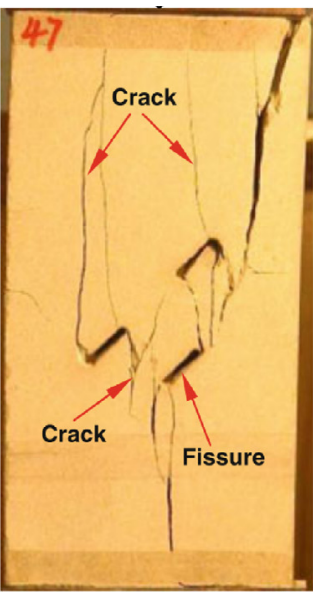

(d)

Fig. 1. Experimental results (a-c) show a time series of modified gypsum specimen under increasing uniaxial compression recovered from highspeed video after Suits et al. [4], with their crack labels subscripted by a number indicating the corresponding propagation sequence. The pictures show: (a) specimen with two initial flaws and partial view of loading apparatus; (b) early-time zoom-in view of wing cracks A-D proceeding from initial flaws; (c) late-time zoom-in view of secondary shear fractures S and coalescent fracture $\mathrm{G}$ in the bridge region between initial flaws, exhibiting the Bobet and Einstein [5] and Bobet [6] type II coalescence pattern; and, (d) after Yang et al. [7], a failed specimen of sandstone with three initial flaws (fissures) exhibiting more complex coalescent crack behavior.

linearly elastic material. Nevertheless, in many geomechanics problems, the geological materials are often subjected to a significant principal stress difference and the materials of interest, such as sedimentary rock, shale, and mudstone, are often inherently heterogeneous and anisotropic. These complexities indicate that the fracture of geological materials under mixed-mode loading is very common. As such, a modeling framework, whether it is based on embedded strong discontinuity or smeared crack approximations, should consider mode mixity in a plausible physical ground that matches the experimental evidence.

Previous experimental works (cf. [8,5,9]), primarily uniaxial and biaxial compression tests in rock, have now established that rock may exhibit a combination of flaw slippage, onset, and propagation of wing (tensile-dominated) and secondary (shear-dominated) cracks, and the coalescence and branching of these cracks, depending on the material properties and the stress conditions, as shown in Fig. 1. Capturing such failure mechanisms faithfully in a numerical model is, nevertheless, not a trivial task. First, the model must be able to capture the distinct energy release rates for the mixed-mode fractures. In other words, the difference in critical energy release rates required to propagate different types of flaws must be quantified [10-14]. Second, the model must be able to replicate the evolving fracture geometrically when the crack propagation direction, as well as direction-dependent kinematics modes, are not known a priori. This task can be further complicated by the coalescence and branching of cracks, leading to even more complex geometries and stress field, which can rapidly increase the essential computational resource [15-21].

To address the first issue, one possible approach is to extend Griffith theory such that (1) cracks grow along the direction that maximizes the fracture dissipation, and that (2) a crack will only grow if and only if the energy release rate reaches a critical level $[22,11]$. This idea is adopted to predict brittle fracture in rock in a 2D setting by Shen and Stephansson [12], where a fracture criterion based on distinct critical energy rates for mode I and mode II is implemented in a displacement discontinuity model to predict the fracture pattern in Reyes and Einstein [8].

Though the adoption of the mixed mode approach may lead to a more realistic prediction on the energy release required to propagate cracks, simulations of evolving cracks remain a challenging problems numerically. While enrichment methods, such as assumed strain method (e.g. [23-25]), extended finite element method (e.g. [26]). and cohesive elements (e.g. [27]) may embed strong discontinuity in the displacement field, the enrichment techniques could become complicated if branching or coalescence occur [16].

A noticeable departure is the recent work by Zhang et al. [14], in which the authors adopt a phase field fracture model to represent cracks with an implicit function and model the mixed-mode fractures with a criterion. The upshot of this approach is that one may leverage the simplicity brought by the regularized geometrical representation of 
cracks. Thus the coalescence and branching of cracks may be modeled without modifying the trial and solution space of the finite element models. Nevertheless, in Xhang et al. [14], the driving forces of the cracks corresponding to mode I and mode II are written as functions of the positive part of the first variant of the infinitesimal strain tensor $\epsilon$ (cf. Eq. (14) in Zhang et al. [14]), i.e.

$$
\mathcal{H}_{I}=\lambda\langle\operatorname{tr}[\epsilon]\rangle^{2},
$$

where the operator $\langle\cdot\rangle=(\cdot+|\cdot|) / 2$ is the Macaulay bracket, and the trace of the positive part of the $\epsilon \cdot \epsilon$ (cf. Eq. (15) in Zhang et al. [14]), i.e.

$$
\mathcal{H}_{I I}=\mu \operatorname{tr}\left[\langle\boldsymbol{\epsilon}\rangle^{2}\right]
$$

where $\lambda>0$ and $\mu>0$ are elastic constants. Obviously, the driving forces in Eqs. (1) and (2) are both isotropic. This can be easily proven by rewriting both $\mathcal{H}_{I}$ and $\mathcal{H}_{I I}$ in terms of principal values of the infinitesimal strain tensor [28,29]. The implication of this isotropic driving force is significant. On the one hand, it greatly simplifies the implementation procedure such that there is no need to determine the propagation direction that maximizes the energy release rate. On the other hand, the isotropy also indicates that this treatment is not compatible to the theoretical work in Sih [22], Nuismer [11], Shen and Stephansson [12], and Shen et al. [13], where the amount of energy release to propagate a crack within a given length is sensitive to both the propagation direction and kinematic modes. It should also be noticed that Griffith fracture mechanics theory is not the only criterion used to predict crack growth for rock. For instance, da Silva and Einstein [30] recently evaluate various stress, strain and energy criteria that predict the onset and propagation of cracks. For instance, da Silva and Einstein [30] concludes that stress- and strain-based crack criteria both lead to better predictions than an energy approach, due to the difficulty in separating tensile and shear behaviors under Griffith theory.

The purpose is this paper is to show that Griffith's energy approach can be formulated via the consistent kinematic modes in a phase field setting. As such, the model is capable of modeling mixed-mode phase field fracture problems and secondary cracks, while allowing one to simulate coalescence and branching without any need for inserting enrichment functions and remeshing. To achieve this goal, we first introduce an algorithm to determine the direction that maximizes energy dissipation at each incremental step. A kinematically consistent model leads to determination of the crack propagation direction locally. The local result is then regularized by application of a diffusive crack model. This allows us to determine the value of the mixed-mode $\mathcal{F}$-criterion from [12] with consistent kinematics. The importance of the consistent kinematics are demonstrated in a number of numerical experiments. The rest of this article is organized as follows. In Section 2, we review the balance principle of the phase field fracture model and provide the extension that leads to the mixed-mode fracture. Then, the search for the crack direction using various energy-derivative approximations is discussed. Numerical examples are then used to demonstrated the capacity of the models in Section 3. Finally, the key findings are summarized in Section 4.

As for notations and symbols, bold-faced letters denote tensors; the symbol '.' denotes a single contraction of adjacent indices of two tensors (e.g. $\boldsymbol{a} \cdot \boldsymbol{b}=a_{i} b_{i}$ or $\boldsymbol{c} \cdot \boldsymbol{d}=c_{i j} d_{j k}$ ); the symbol ' $:$ ' denotes a double contraction of adjacent indices of tensor of rank two or higher (e.g. $\boldsymbol{C}: \boldsymbol{\epsilon}^{e}=C_{i j k l} \epsilon_{k l}^{e}$ ); the symbol ' $\otimes$ ' denotes a juxtaposition of two vectors (e.g. $\boldsymbol{a} \otimes \boldsymbol{b}=a_{i} b_{j}$ ) or two symmetric second order tensors (e.g. $(\boldsymbol{\alpha} \otimes \boldsymbol{\beta})_{i j k l}=\alpha_{i j} \beta_{k l}$ ). Moreover, $(\boldsymbol{\alpha} \oplus \boldsymbol{\beta})_{i j k l}=\alpha_{j l} \beta_{i k}$ and $(\boldsymbol{\alpha} \ominus \boldsymbol{\beta})_{i j k l}=\alpha_{i l} \beta_{j k}$. We also define identity tensors $(\mathbf{1})_{i j}=\delta_{i j},(\boldsymbol{I})_{i j k l}=\left(\delta_{i k} \delta_{j l}+\delta_{i l} \delta_{k j}\right) / 2$, where $\delta_{i j}$ is the Kronecker delta. As for the sign convention, unless otherwise specified, we consider the direction of the tensile stress and dilative pressure as positive.

\section{Methods}

This section is organized as follows. Kinematic assumptions are stated, and the relationship between Griffith theory and phase field fracture model is briefly reviewed. The extension of the phase field fracture model to mixed-mode predictions with consistent kinematic modes is then discussed in detail. In particular, we provide a microforce balancebased derivation for the mixed mode fracture, which leads to a two-field governing equation with displacement and phase field damage as the prime variables. The 1st and 2nd laws of thermodynamics of the mixed-mode phase field fracture model are examined. Our analysis reveals that the necessary condition to prevent spurious healing of the cracks is to enforce the driving force of the mixed-mode fracture being monotonically increasing, which is consistent 
with previous findings in the phase field fracture literature (e.g. [31-33]). Following the formulation of the mixedmode model, we highlight the key features of the implementation. A novel feature critical for the consistent-kinematicmode formulation is the direction search algorithm, required for the local fracture dissipation maximization problem, during the evolution of the phase-field variable. This search algorithm and the corresponding tangent calculation are detailed.

\subsection{Assumptions and modeling approaches}

Consider a brittle material which maintains quasi-static equilibrium, undergoes infinitesimal deformation, and remains in an isothermal state throughout the simulation. As such, the symmetric infinitesimal strain measure is used, thus $\boldsymbol{\epsilon}=\nabla_{s} \boldsymbol{u}$ for $\nabla_{s}(\cdot)=\left(\nabla(\cdot)+\nabla(\cdot)^{\mathrm{T}}\right) / 2$.

Regarding the damage approximation, we adopt a phase field approach to represent cracks $[34,15,31,35]$. In the phase field model, an implicit function is used to indicate the location of the cracks. Let $\Gamma$ be the domain of the crack in a body $\Omega$, then the total crack surface area $A_{\Gamma}$ can be obtained via the integral over crack surface $\Gamma$. As a result, the total crack surface area is approximated as $A_{\Gamma_{d}}$, the volume-integral over body $\Omega$ of the crack surface density $\Gamma_{d}$. In other words,

$$
A_{\Gamma}=\int_{\Gamma} \mathrm{d} A \approx A_{\Gamma_{d}}=\int_{\Omega} \Gamma_{d}(d, \nabla d) \mathrm{d} V,
$$

where the phase field is $d \in[0,1]$, and subscripting $d$ indicates the regularization of a term. The damage phase $d$ varies from 0 in undamaged regions to 1 in fully broken regions [31,36-39]. The corresponding crack density is:

$$
\Gamma_{d}(d, \nabla d)=\frac{1}{2}\left(\frac{d^{2}}{2 l}+\frac{l}{2} \nabla d \cdot \omega \cdot \nabla d\right),
$$

where the length parameter $l>0$ effects regularization, and $\omega$ is a dimensionless symmetric second-order tensor related to the microstructural orientation (cf. [40-42,33,38,43,44]). The introduction of this second order tensor enables one to capture anisotropy of fracture in brittle materials. Thermodynamically, to stop crack healing upon unloading, we require that $\dot{\Gamma}_{d} \geq 0$.

As to mixed-mode fracture, we apply the approach originally proposed in $[12,45]$ where the crack growth criterion depends on two distinctive surface energy release rates/critical fracture energies, i.e. $\mathcal{G}_{I c}$ and $\mathcal{G}_{I I c}$. The mode I and mode II fracture energies rates correspond to opening and shear surface energy dissipates, respectively. To identify opening versus shear energy dissipation, we introduce a strain energy partition depending on the fracture opening (or shearing) direction. This is based upon a microforce balance approach. Locally therefore, a search algorithm is therefore introduced to determine the orientation of the plane (or line in the 2D case) maximizing fracture energy release. While introducing two critical energy release rates have been attempted in [14], to the best knowledge of the authors, this is the first time a phase field fracture model established the driving force consistent with the kinematics of crack growth.

\subsection{Balance principles}

In this work, we formulate the phase field fracture model by balancing the microforce. The phase field models derived from microforce balance can be found in [46,47] for brittle materials, in [43] for capturing brittle-ductile transition of frictional-cohesive materials, Choo and Sun [44] for porous materials with growing crystal, and Na and Sun [38] for anisotropic fracture in crystalline rock. Our new contribution is to introduce a microforce balance that includes a driving force consistent with the kinematic modes on a plane with maximum energy dissipation in the brittle regime. Neglecting the inertial force, the balance of linear momentum reads,

$$
\nabla \cdot \boldsymbol{\sigma}+\rho \boldsymbol{g}=\mathbf{0},
$$

where $\sigma$ is the Cauchy stress, $\rho$ the density, and $\boldsymbol{g}$ the normalized body forces. In this work, the governing equation of the phase field is derived from microforce balance. Hence, we do not have an incremental action functional whose Euler-Lagrange equation becomes the balance of linear momentum and the phase field governing equation. Here, we 
use the classical definition in which the actual stress of the damaged material is related to fictitious (effective) stress of the undamaged material by the degradation function, i.e.

$$
\boldsymbol{\sigma}=g(d) \boldsymbol{\sigma}_{0},
$$

where $g(d)$ is defined in Eq. (19). The stress of undamaged material is defined in Eq. (30). Note that this treatment does not split the tensile and compressive stresses, such that the degradation is applied to both the compressive and tensile components of the stress. This approach is also used in [48,49], and again in [14] for rock-like materials. In reality, the damage due to void growth and extension of micro-cracks may lead to different modulus degradation in tension and compression. A future extension may include distinction between the tensile and compression degradation mechanism similar to [39], where a different degradation function is applied on the compressive stress and the compressive strain energy to reflect the difference in load-bearing capacities. Meanwhile, Wang and Sun [50] has associated the degradation of compression as a result of anti-crack propagation that could be triggered by a higher anti-crack fracture energy to examine the propagation of compaction band. These extensions will be considered in future study but is out of the scope of the current work.

Following the treatment in [46], we postulate the existence of a microforce traction $\xi$, such that the surface microforce $\boldsymbol{\xi} \cdot \hat{\boldsymbol{n}}$ is energy-conjugate to the phase field $d$, for $\hat{\boldsymbol{n}}$ the unit outward normal around a volume. After applications of the divergence theorem and identifying $\nabla \cdot \sigma=-\rho g$, the energy balance reads,

$$
\rho \dot{e}=\sigma: \dot{\boldsymbol{\epsilon}}+\xi \cdot \nabla \dot{d}+(\nabla \cdot \xi) \dot{d} .
$$

where $e$ is the normalized internal energy. Meanwhile, the local microforce balance equation established in [51] reads,

$$
\nabla \cdot \xi+\pi=0
$$

where $\pi$ is a scalar microforce. The phase-conjugate microforce term is partitioned,

$$
\xi=\xi_{I}+\xi_{I I}, \quad \nabla \cdot \xi_{I}=-\pi_{I}, \quad \nabla \cdot \xi_{I I}=-\pi_{I I},
$$

where subscripting $I$ and $I I$ once again indicates quantities conjugate to mode I and mode II energies, based upon a partition of the stored energy function by resolving orthogonal tensors. The idea of phase-field-energy-conjugate force "parts" was recently applied to isotropic/anisotropic energy functions (cf. [39,52]), albeit within the framework of variational fracture, and yet ultimately to yield a similar result. Applying the partition of $\xi$, the dissipation inequality corresponding to Eq. (8) reads:

$$
\mathcal{D}=\sigma: \dot{\boldsymbol{\epsilon}}+\xi_{I} \cdot \nabla \dot{d}+\xi_{I I} \cdot \nabla \dot{d}-\pi_{I} \dot{d}-\pi_{I I} \dot{d}-\dot{\psi} \geq 0 .
$$

where $\mathcal{D}$ is the dissipation, and $\psi$ the stored energy function. The function arguments $\psi(\epsilon, d)$ are assumed.

As mentioned previously, the stored energy function is also partitioned such that,

$$
\psi(\boldsymbol{\epsilon}, d)=\psi_{I}(\boldsymbol{\epsilon}, d)+\psi_{I I}(\boldsymbol{\epsilon}, d)+\psi_{-}(\boldsymbol{\epsilon}) .
$$

Hence:

$$
\dot{\psi}=\frac{\partial \psi}{\partial \boldsymbol{\epsilon}}: \dot{\boldsymbol{\epsilon}}+\left(\frac{\partial \psi_{I}}{\partial d}+\frac{\partial \psi_{I I}}{\partial d}\right) \dot{d}
$$

Applying the derivative expansion, as well as the recognized equality $\sigma=\partial \psi / \partial \epsilon$, to Eq. (10),

$$
\mathcal{D}=\xi_{I} \cdot \nabla \dot{d}-\left(\pi_{I}+\frac{\partial \psi_{I}}{\partial d}\right) \dot{d}+\xi_{I I} \cdot \nabla \dot{d}-\left(\pi_{I I}+\frac{\partial \psi_{I I}}{\partial d}\right) \dot{d} \geq 0 .
$$

We prescribe the identity $\pi^{\mathrm{en}}=\pi-\pi^{\mathrm{dis}}=-\partial \psi / \partial d$, where superscripting en indicates energetic, whereas dis indicates dissipative, microforces. The modal partition becomes

$$
\pi_{I}^{\mathrm{en}}=\pi_{I}-\pi_{I}^{\mathrm{dis}}=-\frac{\partial \psi_{I}}{\partial d}, \quad \pi_{I I}^{\mathrm{en}}=\pi_{I}-\pi_{I I}^{\mathrm{dis}}=-\frac{\partial \psi_{I I}}{\partial d} .
$$

Substituting those identities into the dissipation inequality Eq. (12),

$$
\mathcal{D}=\mathcal{D}_{I}+\mathcal{D}_{I I} \geq 0
$$


where

$$
\mathcal{D}_{I}=\xi_{I} \cdot \nabla \dot{d}-\pi_{I}^{\mathrm{dis}} \dot{d}, \quad \mathcal{D}_{I I}=\xi_{I I} \cdot \nabla \dot{d}-\pi_{I I}^{\mathrm{dis}} \dot{d} .
$$

Heretofore only $\xi$ and $\pi^{\text {dis }}$ remain unidentified. However the energy dissipation is uniformly due to fracture. Hence the change in mode I fracture energy is also the energy dissipation part.

$$
\begin{aligned}
\mathcal{D}_{I} & =\mathcal{G}_{I c} \dot{\Gamma}_{d}=\frac{\mathcal{G}_{I c}}{2}\left(\frac{d}{l} \dot{d}+l \nabla d \cdot \omega \cdot \nabla \dot{d}\right) \\
& =\xi_{I} \cdot \nabla \dot{d}-\pi_{I}^{\text {dis }} \dot{d},
\end{aligned}
$$

where $\mathcal{D}_{I} \geq 0$ if $\dot{\Gamma}_{d} \geq 0$. Matching coefficients in the above equation,

$$
\xi_{I}=\frac{\mathcal{G}_{I c} l}{2} \omega \nabla d, \quad \pi_{I}^{\mathrm{dis}}=-\frac{\mathcal{G}_{I c}}{2}\left(\frac{d}{l}\right) .
$$

Collecting known terms into the mode I microforce balance of Eq. (9),

$$
\pi_{I}=\pi_{I}^{\mathrm{en}}+\pi_{I}^{\mathrm{dis}}=-\frac{\partial \psi_{I}}{\partial d}-\frac{\mathcal{G}_{I c}}{2}\left(\frac{d}{l}\right)=-\nabla \cdot \xi_{I}=-\frac{\mathcal{G}_{I c} l}{2} \nabla \cdot(\boldsymbol{\omega} \cdot \nabla d) .
$$

As an aside to cell-centered finite volume discretization-based readers, $\mathcal{G}_{I c} l \omega$ (and $\mathcal{G}_{I I c} l \omega$ ) should serve as an internal coefficient of the vector Laplacian, if the damage phase is understood to be continuous. Performing the identical procedure for mode II, we arrive at the two-equation system:

$$
\begin{aligned}
-\frac{\mathcal{G}_{I c} l}{2} \nabla \cdot(\omega \cdot \nabla d) & =-\frac{\partial \psi_{I}}{\partial d}-\frac{\mathcal{G}_{I c}}{2}\left(\frac{d}{l}\right), \\
-\frac{\mathcal{G}_{I I c} l}{2} \nabla \cdot(\omega \cdot \nabla d) & =-\frac{\partial \psi_{I I}}{\partial d}-\frac{\mathcal{G}_{I I c}}{2}\left(\frac{d}{l}\right) .
\end{aligned}
$$

The above equations are normalized and summed, to obtain the field equation:

$$
-\frac{1}{\mathcal{G}_{I c} / l} \frac{\partial \psi_{I}}{\partial d}-\frac{1}{\mathcal{G}_{I I c} / l} \frac{\partial \psi_{I I}}{\partial d}-d+l^{2} \nabla \cdot(\omega \cdot \nabla d)=0
$$

In the case of an isotropic material, $\omega$ equals the identity tensor $\mathbf{1}$, but may otherwise incentivize directional-dependent dissipation propensity. The mixed mode stored energy is:

$$
\psi(\boldsymbol{\epsilon}, d)=g_{I}(d) W_{I}(\boldsymbol{\epsilon})+g_{I I}(d) W_{I I}(\boldsymbol{\epsilon})
$$

where $W$ indicates the strain energy of the undamaged fictitious material, and $g_{I}$ and $g_{I I}$ are the degradation functions. Degradation function $g_{I}(d)$ is monotonically decreasing and satisfies: $g_{I}(0)=1, g_{I}(1)=0$, and $g_{I}^{\prime}(1)=0$; the same holds for $g_{I I}(d)$. Substituting the mode I/II parts of $\partial \psi / \partial d$ into Eq. (14),

$$
-g_{I}^{\prime}(d) \frac{W_{I}(\boldsymbol{\epsilon})}{\mathcal{G}_{I c} / l}-g_{I I}^{\prime}(d) \frac{W_{I I}(\boldsymbol{\epsilon})}{\mathcal{G}_{I I c} / l}-d+l^{2} \nabla \cdot(\boldsymbol{\omega} \cdot \nabla d)=0 .
$$

The specific method to enforce crack irreversibility will be discussed in the following section.

\subsection{Energy partition and crack irreversibility}

We prevent the crack healing following the treatment used in [53], such that the global irreversibility constraint of crack evolution can be enforced by ensuring that the local driving force remains non-negative and that the phase field $d$ is monotonically increasing. Assuming that $g(d)=g_{I}(d)=g_{I I}(d)$, Eq. (16) can be rewritten as,

$$
-g^{\prime}(d) \mathcal{F}-d+l^{2} \nabla \cdot(\omega \cdot \nabla d)=0,
$$

where the normalized and nondimensional mixed-mode strain energy reads,

$$
\mathcal{F}=\frac{W_{I}}{\mathcal{G}_{I c} / l}+\frac{W_{I I}}{\mathcal{G}_{I I c} / l}
$$




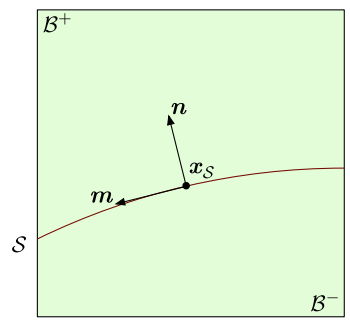

(a)

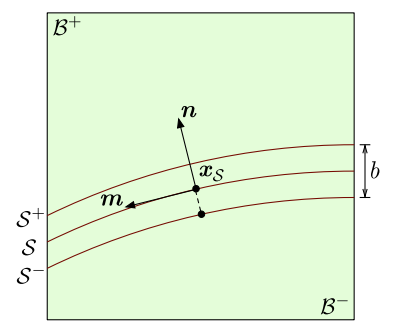

(b)

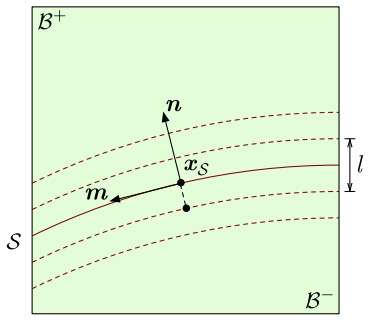

(c)

Fig. 2. Discontinuity models, with (a-b) after Wu and Cervera [56], showing: (a) strong discontinuity model; (b) finite-thickness regularized discontinuity model, for crack width $b$; and, (c) diffusive regularized discontinuity model with the phase field's isolines as dashed.

To halt crack reversibility, just one distinct history or "driving force" function needs be introduced, as evidenced in the following manner. Let the history function $\mathcal{H} \geq 0$ be the pseudo-temporal maximum of the normalized function $\mathcal{F}$. Inserting our definition of $\mathcal{H}$ into Eq. (16):

$$
-g^{\prime}(d) \mathcal{H}-d+l^{2} \nabla \cdot(\omega \cdot \nabla d)=0 .
$$

Eq. (18) is the field equation actually solved for the phase field in below numerical examples. As explained in [54], the thermodynamic consistency of introduced history functions can be checked by considering a spatially homogeneous, isotropic problem where the last term in Eq. (18) vanishes. A monotonically increasing phase field $d$ simply implies that the history function must be monotonically increasing, provided that the derivative of the degradation function remains non-positive. Hence we adopt the same technique to examine the driving force $\mathcal{H}$ for the mixed-mode fracture problem. First, we specify the quadratic degradation function used in below examples as,

$$
g(d)=(1-d)^{2} .
$$

Let $\tilde{\mathcal{H}}=2 \mathcal{H}$ by convention, and for homogeneity $\nabla d=\mathbf{0}$. After substitutions in Eq. (18), we obtain the spatially homogeneous solution by rearranging for $d$, and take its derivative:

$$
d=\frac{\widetilde{\mathcal{H}}}{1+\widetilde{\mathcal{H}}} \in[0,1], \quad \dot{d}=\frac{1}{(1+\widetilde{\mathcal{H}})^{2}} \dot{\widetilde{\mathcal{H}}} \geq 0 .
$$

In other words, if more than one critical energy release rate is used in the $\mathcal{F}$-criterion model, $\dot{\mathcal{H}} \geq 0$ remains that necessary condition to ensure monotonic crack growth and prevent the crack healing after the crack growth, i.e. $\dot{d} \geq 0$.

One simply remedy is to use the maximum value of $\mathcal{F}$ over the time history, rather than the maximum value of the tensile and shear strain energy $W_{I}$ and $W_{I I}$ to formulate the driving force. To summarize:

$$
\mathcal{H}=\max _{\tau \in[0, t]}\{\mathcal{F}\}=\max _{\tau \in[0, t]}\left\{\frac{W_{I}}{\mathcal{G}_{I c} / l}+\frac{W_{I I}}{\mathcal{G}_{I I c} / l}\right\} .
$$

For completeness, we remark that an enhanced correspondence to the original $\mathcal{F}$-criterion can be implemented following the treatment in [54]. In particular, one may restrict the crack growth to initiate above a threshold strain energy by using the following history function for driving force,

$$
\mathcal{H}=\max _{\tau \in[0, t]}\left\{\left\langle\frac{\mathcal{F}}{\mathcal{F}_{c}}-1\right\rangle\right\} .
$$

where $\mathcal{F}_{c}$ is the critical nondimensionalized value of the threshold strain energy. In particular we reference Eq. (19) in [12]: fracture initiates when $\mathcal{F} / \mathcal{F}_{c} \geq 1$ (integrated over the length of an inserted boundary element). Shen and Stephansson [12] also suggest to set the critical toughness ratio to be $\mathcal{G}_{I I c} / \mathcal{G}_{I c} \sim 10-20$ for rocks. However, recent work such as Backers and Stephansson [55] has shown that this critical toughness ratio is not necessarily fixed and may depend on the confining pressure. 


\subsection{Kinematically consistent driving force for phase field}

At this point, we assume that the model is under either 2D plane strain or plane stress condition, such that only the mode I and II fracture energies are considered. To introduce kinematically consistent modal dissipation in 2D cases, we first define an in-plane normal vector describing the opening direction, $\boldsymbol{n}$. Second we define an orthogonal in-plane tangential vector $\boldsymbol{m}$; these are shown in Fig. 2(a), with $\boldsymbol{n} \cdot \boldsymbol{n}=\boldsymbol{m} \cdot \boldsymbol{m}=1$. Together the two vectors $\boldsymbol{n}$ and $\boldsymbol{m}$ form an orthonormal basis spanning $\mathbb{R}^{2}$.

The opening mode is described by the opening-normal $\boldsymbol{n}$ 's dyadic product, and the shearing mode by the $2 \mathrm{D}$ reduced Schmid tensor of $\boldsymbol{n}$ and $\boldsymbol{m}$, respectively,

$$
\boldsymbol{m}_{I}=\boldsymbol{n} \otimes \boldsymbol{n}, \quad \boldsymbol{m}_{I I}=\frac{1}{2}(\boldsymbol{n} \otimes \boldsymbol{m}+\boldsymbol{m} \otimes \boldsymbol{n}),
$$

where $\boldsymbol{m}_{I}: \boldsymbol{m}_{I I}=(\boldsymbol{n} \cdot \boldsymbol{n})(\boldsymbol{n} \cdot \boldsymbol{m})=0$. In turn, we define the opening-mode energy part as

$$
W_{I}=\left\{\begin{array}{cl}
\frac{1}{2}\left(\boldsymbol{\sigma}_{0}: \boldsymbol{m}_{I}\right)\left(\boldsymbol{\epsilon}: \boldsymbol{m}_{I}\right) & \text { if } \boldsymbol{\epsilon}: \boldsymbol{m}_{I} \geq 0 \\
0 & \text { otherwise. }
\end{array}\right.
$$

We define the mode I strain as $\epsilon_{I}=\boldsymbol{\epsilon}: \boldsymbol{m}_{I}$, which can be interpreted as the regularized (homogenized) mode I opening. Similarly, the shearing-mode energy part reads,

$$
W_{I I}=\frac{1}{2}\left(\sigma_{0}: \boldsymbol{m}_{I I}\right)\left(\boldsymbol{\epsilon}: \boldsymbol{m}_{I I}\right),
$$

where the effective stress $\sigma_{0}(\boldsymbol{\epsilon})$ depends on the strain, but is not necessarily co-axial with the elastic strain tensor. In Eq. (24), we impose the restriction $\boldsymbol{\epsilon}: \boldsymbol{m}_{I} \geq 0$ for the reasons: (1) practically to stop fracture in compressive zones, and (2) conceptually to enforce kinematically consistent mode I dissipation in the opening-mode only, Fig. 2(b-c). The coordinate $\boldsymbol{x}_{\mathcal{S}}$ prescribed by the crack normal $\boldsymbol{n}$ is outside the scope of this study.

Note that the above implies an orthogonal if incomplete partition of the strain energy. The idea is simple: $\boldsymbol{n}$ approximates the opening-mode direction, such that $\boldsymbol{m}$ describes the in-plane direction of the fracture surface. As such, $W_{I}$ is strain energy due to tensile stresses resolved in the opening-mode direction. Similarly $W_{I I}$ is the energy resulting from shearing along the fracture surface. In plane stress or plane strain, both $W_{I}$ and $W_{I I}$ are uniquely defined by $\boldsymbol{m}=\boldsymbol{e}_{3} \times \boldsymbol{n}$, where $\boldsymbol{e}_{3}$ is the out-of-plane vector. Being that the fracture-opening direction is approximated kinematically (as $\boldsymbol{n}$ ), the energies of the tensile versus shear stresses and strains are the product of the magnitudes of orthogonal vectors.

By the principles we later describe (viz. use of an operator split), $\sigma_{0}$ and $\epsilon$ are fixed prior to converging $d$. The strain energy partition is then determined as

$$
\boldsymbol{n}=\left.\arg \max _{\boldsymbol{n}}\{\mathcal{F}(\boldsymbol{n})\}\right|_{\boldsymbol{\epsilon}} .
$$

For an isotropic material, in principle, $\boldsymbol{n}$ 's orientation can be determined analytically from $\boldsymbol{\epsilon}$ 's eigenvalues and vectors alone. For an anisotropic material, where the directions of the principle stresses and straines may not coincide, that generalization is untrue. Just such a material model is introduced in below section.

\subsection{Driving force for transversely isotropic materials}

We adopt the form of energy functional in [33] to replicate the elastic responses of a transversely isotropic material. Here we introduce only a marginal modification to the strain energy functional. This modification is appropriate for certain rock-like materials, if they exhibit enhanced compliance in the out of (transversely isotropic) plane direction. For example, the relative stiffnesses of shale rock anisotropy are discussed in [57,58]. To do this, we define:

$$
W_{0}(\boldsymbol{\epsilon})=W_{I}(\boldsymbol{\epsilon})+W_{I I}(\boldsymbol{\epsilon})+W_{-}(\boldsymbol{\epsilon}),
$$

for the anisotropic effective energy functional $W_{0}$,

$$
W_{0}(\boldsymbol{\epsilon})=\frac{\lambda}{2}(\mathbf{1}: \boldsymbol{\epsilon})^{2}+\mu \boldsymbol{\epsilon}: \boldsymbol{\epsilon}+\frac{\phi}{2}(\boldsymbol{\phi}: \boldsymbol{\epsilon})^{2}+\frac{\chi}{2}(\chi: \boldsymbol{\epsilon})^{2},
$$


where the additional elastic constants are $\phi>0$ and $\chi>0$, and the microstructural, second-order tensors are:

$$
\phi=l \otimes l, \quad \chi=1-\phi,
$$

where $\boldsymbol{l}$ is the out-of-isotropic-plane direction requiring $\boldsymbol{l} \cdot \boldsymbol{l}=1$.

Similarly, the surface energy diffusion tensor is defined by the structural tensors as:

$$
\boldsymbol{\omega}=\mathbf{1}+\alpha \boldsymbol{\phi}+\beta \chi,
$$

where $\alpha \gg 0$ penalizes damage diffusion on planes normal to $l$; in contrast $\beta \gg 0$ encourages damage diffusion on planes normal to $l$. In order that $\omega$ be positive semi-definite, $\alpha \geq-1$ and $\beta \geq-1$.

Finally, with the stored energy functional now being defined, we specify the effective stress

$$
\boldsymbol{\sigma}_{0}=\frac{\partial W_{0}}{\partial \boldsymbol{\epsilon}}=\lambda(\mathbf{1}: \boldsymbol{\epsilon}) \mathbf{1}+2 \mu \boldsymbol{\epsilon}+\phi(\boldsymbol{\phi}: \boldsymbol{\epsilon}) \boldsymbol{\phi}+\chi(\chi: \boldsymbol{\epsilon}) \chi,
$$

and by resolving $\sigma_{0}$ in Eqs. (24) and (25), then renotating $W_{I}=\left(\boldsymbol{\sigma}_{0}: \boldsymbol{m}_{I}\right)\left\langle\boldsymbol{\epsilon}: \boldsymbol{m}_{I}\right\rangle / 2$,

$$
W_{I}(\boldsymbol{\epsilon})=\left[\frac{\lambda}{2}(\boldsymbol{\epsilon}: \mathbf{1})+\mu\left(\boldsymbol{\epsilon}: \boldsymbol{m}_{I}\right)+\frac{\phi}{2}(\boldsymbol{\epsilon}: \boldsymbol{\phi})\left(\boldsymbol{\phi}: \boldsymbol{m}_{I}\right)+\frac{\chi}{2}(\boldsymbol{\epsilon}: \chi)\left(\chi: \boldsymbol{m}_{I}\right)\right]\left\langle\boldsymbol{\epsilon}: \boldsymbol{m}_{I}\right\rangle,
$$

and the shear energy is

$$
W_{I I}(\boldsymbol{\epsilon})=\left[\mu\left(\boldsymbol{\epsilon}: \boldsymbol{m}_{I I}\right)+\frac{\phi}{2}(\boldsymbol{\epsilon}: \boldsymbol{\phi})\left(\boldsymbol{\phi}: \boldsymbol{m}_{I I}\right)+\frac{\chi}{2}(\boldsymbol{\epsilon}: \chi)\left(\chi: \boldsymbol{m}_{I I}\right)\right]\left(\boldsymbol{\epsilon}: \boldsymbol{m}_{I I}\right),
$$

where we have used $\mathbf{1}=\boldsymbol{n} \otimes \boldsymbol{n}+\boldsymbol{m} \otimes \boldsymbol{m}+\boldsymbol{e}_{3} \otimes \boldsymbol{e}_{3}$, thus $\mathbf{1}: \boldsymbol{m}_{I}=1$ and $\mathbf{1}: \boldsymbol{m}_{I I}=0$.

In Eq. (30), we expect nonzero $\phi$ only if $\chi=0$, and visa versa. Furthermore, if the stiffer directions are also more brittle, then fractures may propagate preferentially in directions of lower initial elastic compliance. If this happens, then either: $\phi>0$ and $\alpha>0$; or, $\chi>0$ and $\beta>0$ must hold. The latter combination would be more appropriate for the characterization of macroscopic effective properties of bedded or layered materials, such as shale rock, as indicated in [59].

In the same vein, the second derivative of $W_{0}$ in $\epsilon$ does not elicit linearly independent fourth-order tensors spanning the full space of transversely isotropic stiffness tensors (cf. [60]). To conduct the below-contained numerical experiments, whilst simultaneously approximating rock anisotropy, the following heuristic is adopted: $(\lambda+\chi) / \lambda \approx$ $E / E^{*}$, for $E / E^{*}$ the ratio of the in-plane over the out-of-plane Young's moduli. For the shale rock type for instance, $\chi \approx\left(E / E^{*}-1\right) \lambda$ where $E / E^{*}$ is $\sim 2$.

\subsection{Direction search algorithm}

To obtain the correct driving force, one must first determine the orientation of a plane in which the corresponding mode I and II kinematic modes maximize the energy dissipation. In the context of eigenfracture or element-erosion models (cf. [61-63]), this orientation is not directly determined, but the energy loss for each possible eroded configuration is compared. The eroded element that leads to maximum energy dissipation is chosen to propagate the crack. In [37] and [64], the choice for crack propagation direction remains finite, but the crack is captured as an embedded strong discontinuity. In this work, we leverage one of the most important advantages of using implicit function to represent crack geometry: the ability to ensure crack growth in arbitrary directions. The trade-off is that (1) the crack is not represented explicitly as a displacement jump and (2) the mesh must be sufficiently fine such that the implicit function has sufficient resolution to represent the interface. This trade-off could be a sensible choice for the mixed-mode fracture simulations due to the inherent anisotropy of the materials and the anisotropy induced by the multiple crack growth mechanisms. Nevertheless, due to the introduction of the additional critical energy release rate, a search algorithm must be used to determine the orientation that maximizes energy dissipation. To conduct the direction search in 2D domain, we parameterize the normal vector $\boldsymbol{n}$ of $\mathcal{F}(\boldsymbol{n})$ such that the orientation can be described by a single parameter $\theta$,

$$
\boldsymbol{n}(\theta)=[\cos \theta, \sin \theta, 0], \quad \boldsymbol{m}(\theta)=\boldsymbol{e}_{3} \times \boldsymbol{n}=[-\sin \theta, \cos \theta, 0],
$$

where $\theta=\operatorname{acos}\left(\boldsymbol{n} \cdot \boldsymbol{e}_{1}\right)$ is the angle between the normal vector $\boldsymbol{n}, \boldsymbol{e}_{1}=[1,0,0]$, and $\boldsymbol{e}_{2}=[0,1,0]$. Together coordinate directions $\boldsymbol{e}_{1}, \boldsymbol{e}_{2}$, and $\boldsymbol{e}_{3}$ span $\mathbb{R}^{3}$. Furthermore, as only the dyadic products of $\boldsymbol{n}$ and $\boldsymbol{m}$ are used to resolve $W_{I}$ and $W_{I I}, \mathcal{F}(\theta)=\mathcal{F}(\theta+\pi)$, the search is conducted on $\theta \in[0, \pi)$. 


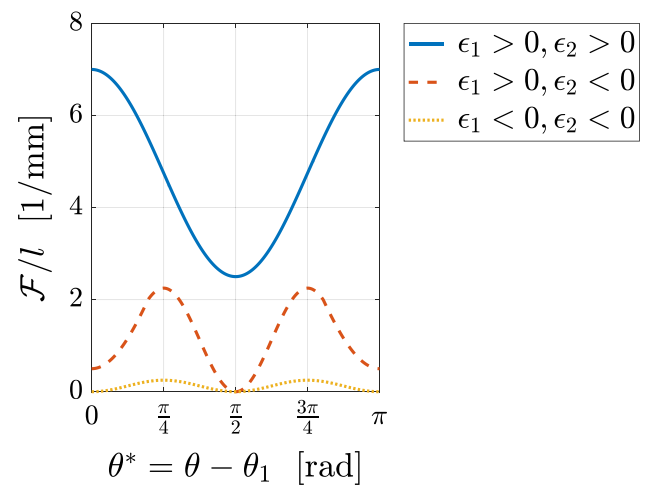

(a) $\mathcal{G}_{\text {IIc }} / \mathcal{G}_{I c}=1$, varying $\epsilon$.

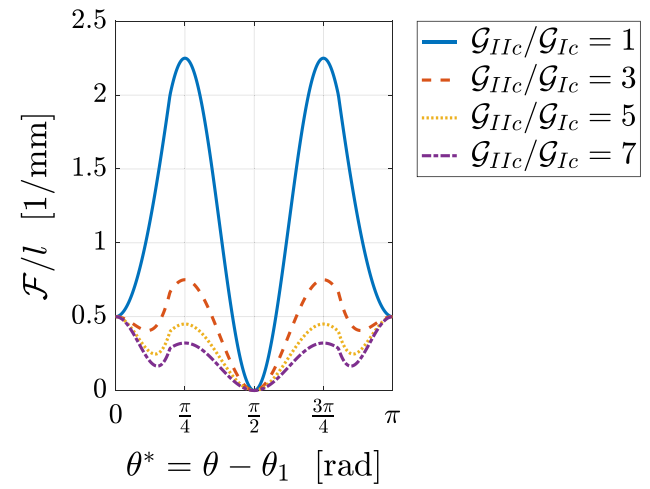

(b) Fixed $\epsilon$, varying $\mathcal{G}_{\text {IIc }} / \mathcal{G}_{\text {Ic }}$

Fig. 3. $\mathcal{F} / l$ for distinct in-plane $\boldsymbol{\epsilon}$-eigenvalues, $\theta^{*}$ is the angle between the direction $\boldsymbol{n}$ and the eigenvector associated with the greatest principal strain, and $\theta_{1}$ is the angle between that eigenvector and the coordinate direction $\boldsymbol{e}_{1}$.

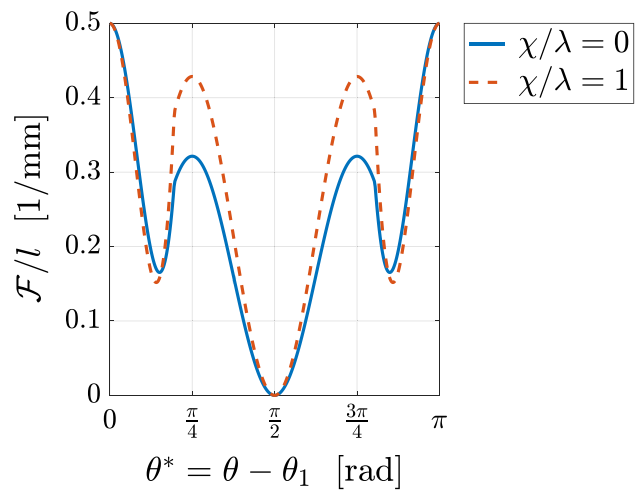

(a) Isotropy vs. anisotropy for $\theta_{l}^{*}=0$.

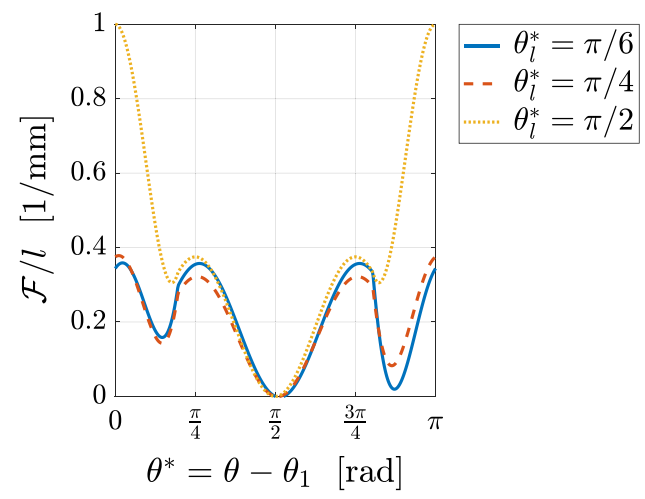

(b) $\chi / \lambda=1$ varying $\theta_{l}^{*}(l)$.

Fig. 4. $\mathcal{F} / l$ for transverse isotropic materials with critical fracture energy ratio $\mathcal{G}_{I I c} / \mathcal{G}_{I c}=7$. As shown in (b), anisotropy perturbs the local maxima and minima from $\theta^{*}=n \pi / 4 \mid n \in\{0,1,2,3\}$, because stress's and strain's spectral directions are not coaxial.

Examples of the calculation via this $\theta$-parameterizations of $\mathcal{F}$ are shown in Fig. 3. For a given set of elastic material parameters and strain, the driving force is calculated as a function of angle $\theta$, following the same treatment in [12] through [65]. As a result, $\mathcal{F}$ depends on the strain state per Fig. 3(a), as well as the ratio $\mathcal{G}_{I I c} / \mathcal{G}_{I c}$. The material parameters are $\lambda=\mu=40.0 \mathrm{kN} / \mathrm{mm}^{2}$, and $\mathcal{G}_{I c}=1.0 \times 10^{-3} \mathrm{kN} / \mathrm{mm}$, with generally the principal strains $\epsilon_{1}=0.005$ and $\epsilon_{2}=-0.01$, and the corresponding eigenvectors equal to $\boldsymbol{n}_{1}=[1,0,0]$ and $\boldsymbol{n}_{2}=[0,1,0]$, respectively. Recall that the spectral form of the strain tensor reads,

$$
\boldsymbol{\epsilon}=\sum_{A=1}^{\operatorname{dim}} \epsilon_{A} \boldsymbol{n}_{A} \otimes \boldsymbol{n}_{A} .
$$

where $\operatorname{dim}$ is the dimension of the domain. As to notation, superscripting $*$ indicates: $\theta^{*}=\theta-\theta_{1}$ for $\theta_{1}$ the direction of the most positive principal strain, and $\mathcal{F} / l$ a value not normalized by the phase field length parameter $l$. For a given plane strain state, which leads to one positive and one negative principal stress in an isotropic material, we observe that the $\mathcal{F}$ is maximized at $\theta^{*}=\pi / 4$ when $\mathcal{G}_{I I c} / \mathcal{G}_{I c}=1$, Fig. 3(b). However, when $\mathcal{G}_{I I c} / \mathcal{G}_{I c}=7, \mathcal{F}$ is maximized at $\theta^{*}=0$. This change indicates a transition from primary shearing- to opening-mode fracture, as the ratio $\mathcal{G}_{\text {IIc }} / \mathcal{G}_{\text {Ic }}$ increases from 1 to 7 .

In cases where the elastic response of the material exhibits transverse isotropy, the material symmetry may affect the orientation of the plane that maximizes energy dissipation. Fig. 4 shows the results of the numerical experiments 
performed on transversely isotropic materials, as described mechanically by Eq. (30). Given the same strain and the ratio $\mathcal{G}_{I I c} / \mathcal{G}_{I c}=7$, we first raise $\chi$ from 0 to $\chi=\lambda$ in Fig. 4(a) and change the orientation of the plane of isotropy of the elasticity tensor in Fig. 4(b). In the numerical example shown in Fig. 4(a), we observe that the anisotropy of the elasticity may change the driving force as expected. In the numerical example shown in Fig. 4(b), the angle between the normal vector of the plane of the isotropy and $\boldsymbol{e}_{1}$ varies from $\pi / 6$ to $\pi / 2$. This latter result indicates not only that the anisotropy of the elasticity can alter the dominant mode of fracture, but also breaks the symmetry of the driving force profile against $\theta^{*}$.

Subsequent to the parameterization, the driving force becomes a function of the angle $\theta$ for a given strain state. As such, we may use a gradient based optimization procedure to find all the local maxima such that $d \mathcal{F} / d \theta=0$ and $d^{2} \mathcal{F} / d \theta^{2}<0$. The orientation corresponding to the largest local maximum will be used to compute the driving force. For the unknown $x^{k}=\theta$ at the procedure's $k$-th iteration, the integration point residual vector and local tangent operator of the optimization problem are:

$$
r\left(x^{k}\right)=\frac{\mathrm{d} \mathcal{F}}{\mathrm{d} \theta}, \quad r^{\prime}\left(x^{k}\right)=\frac{\mathrm{d}}{\mathrm{d} \theta}\left(\frac{\mathrm{d} \mathcal{F}}{\mathrm{d} \theta}\right) .
$$

The local maxima can be determined by finding all the roots of the one-parameter equation $d \mathcal{F} / \mathrm{d} \theta=0$. We then select the maximum root of $\mathrm{d} \mathcal{F} / \mathrm{d} \theta=0$ to determine the orientation. Since gradient-based optimization is used $\mathrm{d} \mathcal{F} / \mathrm{d} \theta$ and its derivative must be computed. This can be done by obtaining the exact expression of $\mathrm{d} \mathcal{F} / \mathrm{d} \theta$ and $\mathrm{d}^{2} \mathcal{F} / \mathrm{d} \theta^{2}$.

However, a simpler approach is to use numerical approximations, such as central difference (CD) or complex stepping (CS), the latter having been introduced by Lyness and Moler [66]. The approximated derivatives of $\mathcal{F}$ obtained from the CD and CS methods read

$$
\frac{\mathrm{d} \mathcal{F}}{\mathrm{d} \theta} \approx \frac{\mathcal{F}(\theta+h)-\mathcal{F}(\theta-h)}{2 h}, \quad \frac{\mathrm{d} \mathcal{F}}{\mathrm{d} \theta} \approx \frac{\mathfrak{I}\{\mathcal{F}(\theta+i h)\}}{h},
$$

where $h$ is a small value, $\Im\{\cdot\}$ is the imaginary part of $\cdot$, and $i=\sqrt{-1}$. CS has been of recent interest to material modelers (cf. $[67,68]$ ) to approximate tensorial derivatives relating to the residual of field equations. As a minor caveat, regarding the CS approximation, the condition in Eq. (24) is evaluated as $\Re\left\{\boldsymbol{\epsilon}: \boldsymbol{m}_{I}\right\} \geq 0$, where $\Re\{\cdot\}$ is the real part of $\cdot$

Subsequent to the spectral decomposition of the strain, we determine from the opening- and material-directional cosines,

$$
\theta_{1}=\arccos \left(\boldsymbol{n}_{1} \cdot \boldsymbol{e}_{1}\right), \quad \theta_{\boldsymbol{l}}=\arccos \left(\boldsymbol{l} \cdot \boldsymbol{e}_{1}\right),
$$

where $\boldsymbol{n}_{1}$ is the direction of the most positive principal strain $\epsilon_{1}$. As noted, because $\mathcal{F}$ exhibits more-than-one local maximum, gradient-based optimization is applied sequentially. Accordingly the several applications employ $\theta_{1}+n \pi / 4 \mid n \in\{0,1,2,3\}$ sequentially as the initial guess for $\theta$. If the material is anisotropic, then microstructural directions are additionally accounted for.

Discontinuities in $\mathrm{d} \mathcal{F} / \mathrm{d} \theta$ may arise from resolving $\epsilon_{I}$ in Eq. (24), where from fracture dissipation in mode $I$ is restricted to crack opening. A discontinuity at $\theta_{0}$ is identifiable given $\epsilon_{I}=0$,

$$
\theta_{0}^{*}=\theta_{0}-\theta_{1}=\arctan \sqrt{\left|\epsilon_{1} / \epsilon_{2}\right|},
$$

for $\epsilon_{1}$ and $\epsilon_{2}$ the most and least positive principal strains, Fig. 5. For the reason of discontinuity, and the associated marginal accuracy improvements, we have designed the system to: (1) employ the complex-step approximation; and, (2) separate the maximization problem into two analytic functions, and these are given by

$$
\text { case (i) }: \frac{1}{2}\left(\boldsymbol{\sigma}_{0}: \boldsymbol{m}_{I}\right)\left(\boldsymbol{\epsilon}: \boldsymbol{m}_{I}\right)+W_{I I}, \quad \text { and case (ii) }: W_{I I} \text {. }
$$

The first analytic function's maxima are discarded if corresponding $\epsilon_{I}<0$. As a backstop near $\epsilon_{I}=0, \theta$ approaching the derivative discontinuity are also considered. Separately, both positive and negative perturbations of $\mathcal{F}(\theta)$ by $h$ around $\theta_{0}$ are evaluated. The symmetric root is then also perturbed and checked as maximizers, see vertical line $\pi-\theta_{0}^{*}=\pi-\left(\theta_{0}-\theta_{1}\right)$ in Fig. 5. 


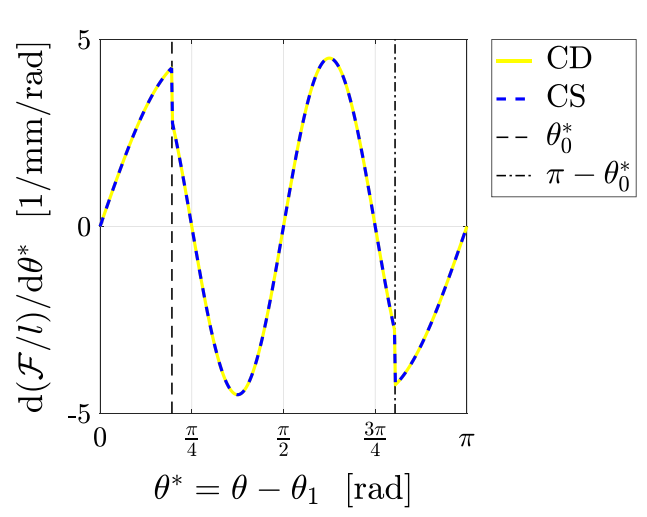

(a) Distinct $\epsilon$-eigenvalues.

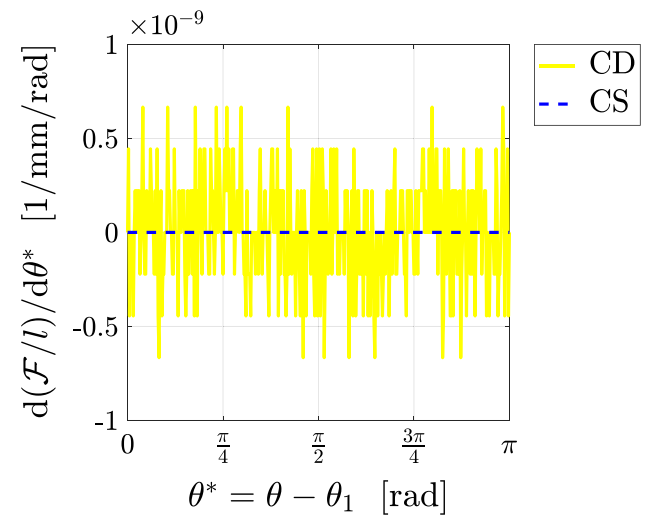

(b) Repeated $\boldsymbol{\epsilon}$-eigenvalue.

Fig. 5. Central difference and complex step $\mathcal{F}$-derivative approximations, contrasting distinct and repeated in-plane strain eigenvalues, with (a) showing derivative jumps at $\theta_{0}^{*}$ and $\pi-\theta_{0}^{*}$ as dashed vertical lines. The ratio between the two critical energy release rates is $\mathcal{G}_{I I c} / \mathcal{G}_{I c}=1$.

\subsection{Galerkin discretization}

With the local problem solved for $\theta, \boldsymbol{n}$, and $\mathcal{F}$, finite elements are used to discretize the spatial domain. Boundary conditions on body $\mathcal{B}$ with surface $\partial \mathcal{B}$ are specified, i.e.

$$
\begin{array}{rll}
\boldsymbol{u}=\hat{\boldsymbol{u}} & \text { on } & \partial \mathcal{B}_{\boldsymbol{u}}, \\
\boldsymbol{\sigma} \cdot \hat{\boldsymbol{n}}=\hat{\boldsymbol{t}} & \text { on } & \partial \mathcal{B}_{t}, \\
\nabla d \cdot \hat{\boldsymbol{n}}=0 & \text { on } & \partial \mathcal{B},
\end{array}
$$

where $\hat{\boldsymbol{u}}$ is the boundary displacement, $\hat{\boldsymbol{t}}$ the resolved stress, and $\hat{\boldsymbol{n}}$ refer to the outward-pointing unit normal. The trial function spaces are posited,

$$
\begin{aligned}
& \mathcal{S}_{u}=\left\{\boldsymbol{u} \mid \boldsymbol{u} \in H^{1}, \boldsymbol{u}=\hat{\boldsymbol{u}} \text { on } \partial \mathcal{B}_{\boldsymbol{u}}\right\}, \\
& \mathcal{S}_{d}=\left\{d \mid d \in H^{1}\right\},
\end{aligned}
$$

complimented by the spaces of test functions $\eta$ and $\phi$,

$$
\begin{aligned}
& \mathcal{V}_{u}=\left\{\boldsymbol{\eta} \mid \boldsymbol{\eta} \in H^{1}, \boldsymbol{\eta}=\mathbf{0} \text { on } \partial \mathcal{B}_{u}\right\}, \\
& \mathcal{V}_{d}=\left\{\phi \mid \phi \in H^{1}\right\} .
\end{aligned}
$$

where $H^{1}$ is the Sobolev space of degree 1. Weak forms of Eqs. (5) and (16) are achieved by Green's theorem and $\sigma^{\prime}$ s symmetry such that

$$
\int_{\mathcal{B}} \nabla_{s} \boldsymbol{\eta}: \boldsymbol{\sigma} \mathrm{d} V=\int_{\mathcal{B}} \boldsymbol{\eta} \cdot \rho \boldsymbol{g} \mathrm{d} V+\int_{\partial \mathcal{B}_{t}} \boldsymbol{\eta} \cdot \hat{\boldsymbol{t}} \mathrm{d} A
$$

and similarly for Eq. (16),

$$
\int_{\mathcal{B}} \phi g^{\prime}(d) \mathcal{H} \mathrm{d} V+\int_{\mathcal{B}}\left(\phi d+l^{2} \nabla \phi \cdot \omega \cdot \nabla d\right) \mathrm{d} V=0 .
$$

The spatial domain is discretized with standard low-order quadrilateral finite elements. The implementation of the spatial discretization is done using the finite element library deal . ii [69], whereas the implicit nonlinear PDE solver, including the assembly procedure of the residuals and the corresponding tangents, and the Newton-Raphson scheme are modified from the software code base geocentric [70-72,38,43,44].

The mixed-mode fracture model is implemented in a non-iterative operator-split algorithm in which the incremental displacement and phase field are updated sequentially. As pointed out in previous work, such as Miehe et al. [73] and Wheeler et al. [74], the non-iterative operator-split solver is faster than the monolithic counterpart. Nevertheless, 


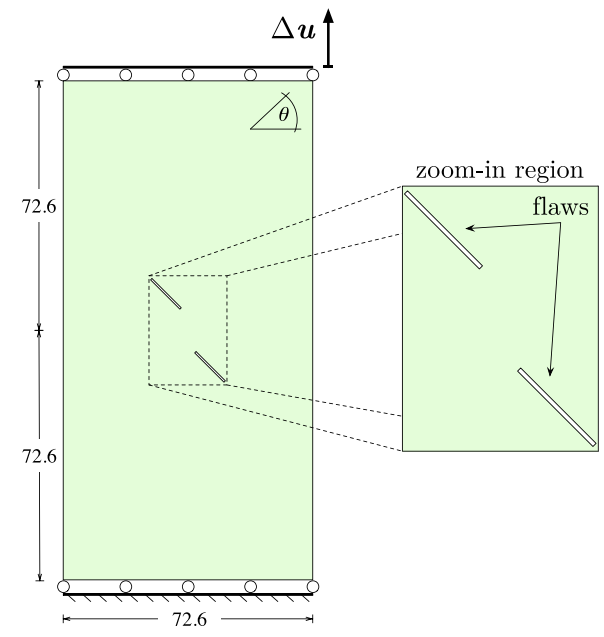

(a) Setup of the boundary-value problem.

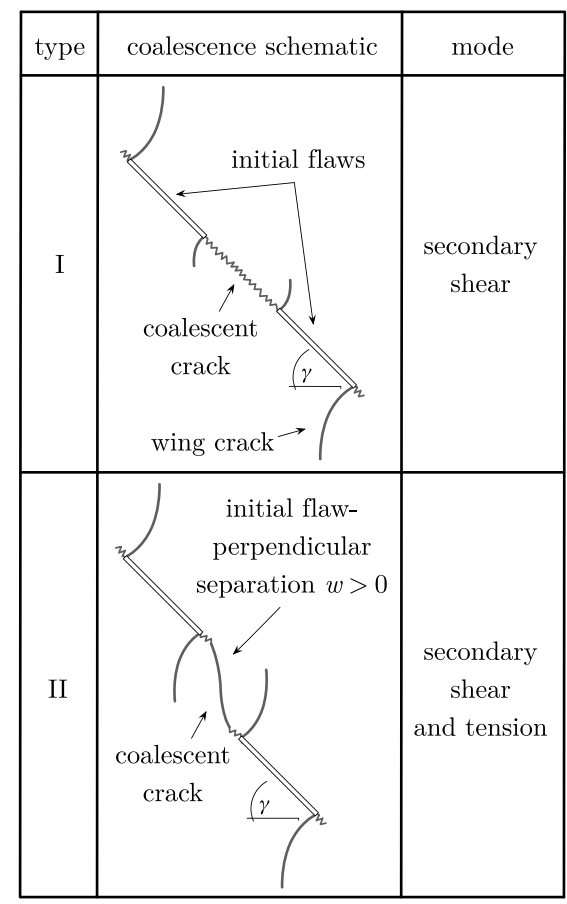

(b) Relevant mixed-mode crack types.

Fig. 6. Geometry of the numerical specimen for mixed-mode fracture simulations, with (b) schematizing experimental results for the same geometry abridged from the extended typology of Bobet [6], in which the authors attribute the zig-zag crack pattern to shear-induced fracture, viz. with a rough crack surface coated with crushed gypsum.

the incremental step must be sufficiently small to ensure that the global residuals remain below the numerical tolerance. Since the details of the operator-split solver has been described in great detail in previous work, we omit the details.

\section{Numerical examples}

The following boundary-value problems are used to showcase the capacity of the proposed model to replicate mixed-mode crack growth. Fracture mode mixity may lead to (1) wing cracks and (2) secondary shear-dominant and mixed shear-dominant cracks. These are experimental results of Bobet and Einstein [75], modeled numerically with boundary elements by Shen and Stephansson [12] and a phase-field crack approximation by Zhang et al. [14], also assuming distinct modal fracture energies. The problems describe two initial flaws situated relatively closely together, with experimental results suggesting complex mixed-mode inter-flaw coalescent fracture. Unless otherwise specified, we set $\phi=\chi=0$ and $\alpha=\beta=0$ reducing to isotropy, assume plane strain, and dimensions on diagrams are in $\mathrm{mm}$.

The external boundaries are fixed in dimension, and the rectangular internal, initial flaws are fixed in length at $12.7 \mathrm{~mm}$ and width at $0.1 \mathrm{~mm}$. The flaws' angle relative to the horizontal axis is $\gamma=\pi / 4$, their closure is $c=12.7$ $\mathrm{mm}$, and they are parameterized by their separation $w$, Fig. 6(a). Parameter $\gamma=\pi / 4$ was taken for two reasons. First, the numerical specimen with $\pi / 4$ flaw angle has been used in the literature as benchmark for the energy argument-based Displacement Discontinuity Method (DDM) model of Shen and Stephansson [12] and Shen [45], the mixed-mode phase field fracture model in [14], and the stress-criterion-based crack propagation model of Bobet and Einstein [75] and Bobet [6]. Hence, this setup is convenient for comparison purposes. Second, numerical specimens with this flaw orientation may lead to the development of mixed-mode cracks, as demonstrated in the aforementioned literature. Hence using this same setup helps us showcase the effect of the elastic anisotropy on crack propagations, when anisotropic materials with different transversely isotropic planes are used in simulations. An abbreviation of 


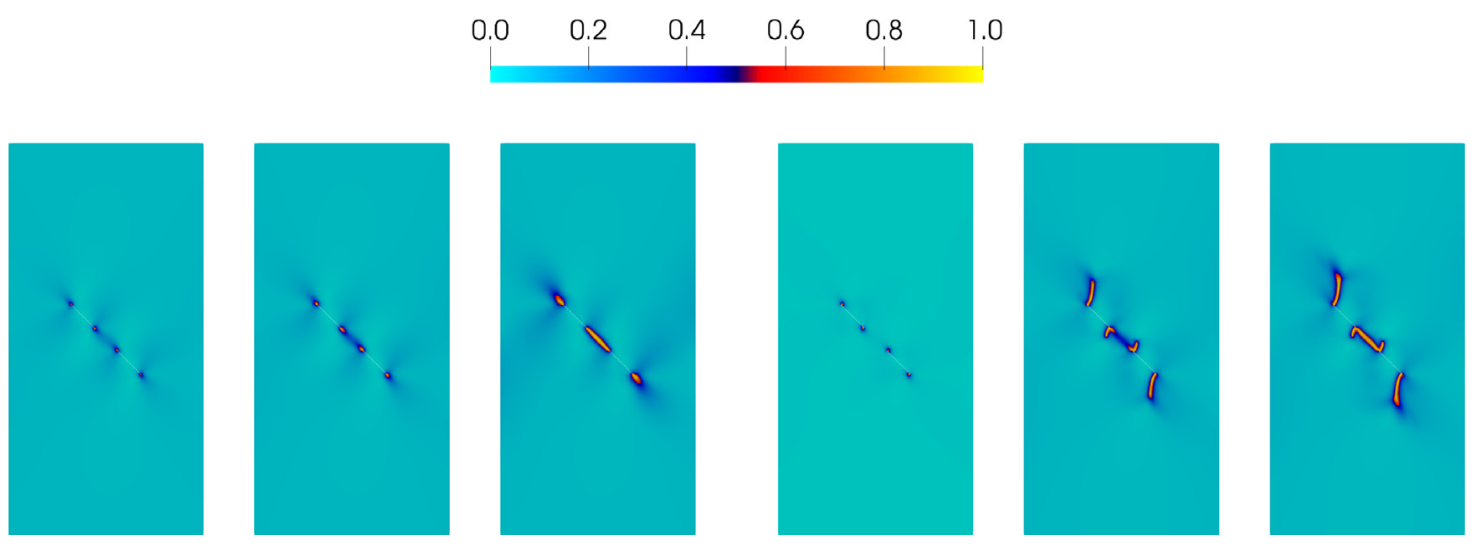

(a) $\mathcal{G}_{\text {IIc }} / \mathcal{G}_{\text {Ic }}=1$, at $u_{2}=190,195,199 \times-10^{-4} \mathrm{~mm}$.

(b) $\mathcal{G}_{I I c} / \mathcal{G}_{I c}=7$, at $u_{2}=265,512,514 \times-10^{-4} \mathrm{~mm}$.

Fig. 7. Isotropic mixed-mode case: phase field $d$ for separation $w=0 \mathrm{~mm}$, without significant topological change in closure crack geometry between critical fracture energy ratios $\mathcal{G}_{I I c} / \mathcal{G}_{I c}=3$ and 7.

experimentally recovered crack-coalescence pattern typology is presented in Fig. 6(b), as provided by Bobet and Einstein [75,5] and expanded in [6]. As indicated by Bobet and Einstein [5], this abridged typology is suitable for molded gypsum specimens compressed uniaxially or at low confining stresses.

The material parameters correspond to molded gypsum: $\lambda=3.08 \mathrm{kN} / \mathrm{mm}^{2}$ and $\mu=2.42 \mathrm{kN} / \mathrm{mm}^{2}$ exactly from [8,12], and $\mathcal{G}_{I c}=50.0 \times 10^{-6} \mathrm{kN} / \mathrm{mm}$ reflecting Shen and Stephansson [12]. The length scale parameters are $l=0.5942 \mathrm{~mm}$, with a near-fracture fine mesh employed, in order to capture the sharp phase field gradient. Our heuristic observations from the numerical experiments indicate that insufficiently small $l$ and near-flaw mesh element lengths can sometimes suppress secondary cracks.

For this reason, we use an element characteristic length of $0.06 \mathrm{~mm}$ in the flaw-tip region, $0.25 \mathrm{~mm}$ in the flaw region, coarsening to $0.5 \mathrm{~mm}$ near the domain external boundaries, with refinement maintained within about $c / 2$ of the flaws. The left and right external boundaries are both traction free. On the bottom boundary $\Delta u_{2}=0 \mathrm{~mm}$ for all time steps, with the $x_{2}$-direction traction free, i.e. fixed normal displacement with zero shear. On the top boundary we prescribe $\Delta u_{1}=-5.0 \times 10^{-4} \mathrm{~mm}$, and the $x_{2}$-direction is traction free. The initial flaws are considered traction free.

\subsection{Isotropic coalescent cases}

The parametric study of $\mathcal{G}_{I I c}$ and the various closure geometries of the initial flaws are shown in Figs. 7 and 8. If $\mathcal{G}_{I I c} / \mathcal{G}_{I c}$ is less than a necessary threshold value, no wing cracks are apparent, and the specimens exhibit similar fracture patterns to those materials with $\mathcal{G}_{I I c} / \mathcal{G}_{I c}=1$. If the ratio exceeds this threshold, wing cracks initiate and propagate; subsequently secondary cracks initiate in mixed-strain zones. Finally, as opposed to the relatively stable growth of the tensile-dominant wing cracks (where present), the shear-dominant fractures coalesce brutally: over the course of about 5 boundary displacement-driven load increments, as shown in Fig. 9, applying an operator-split algorithm to advance the simulation. Here we follow the terminology in [76] which refers to a brutal crack propagation as the crack growth that occurs while dissipation increases rapidly and leads to a sudden dip in total free energy. Strictly speaking, brutal cracking may lead to substantial inertial effect which cannot be completely accounted for in a quasi-static framework, according to Negri and Ortner [77]. Accommodating the dynamic phenomenon is out the scope of this study but will be considered in the future. However when separation $w>0$, the flaw-misalignment of the secondary cracks occasions turning during the coalescence. Of significance overall, the Macaulay bracket in Eq. (31) usefully doubles to eliminate mode I fracture in uniaxial compaction: a welcome by-product of kinematic consistency.

The wing cracks' initiation and propagation directions are least sensitive to the ratio $\mathcal{G}_{I I c} / \mathcal{G}_{I c}$, above $\mathcal{G}_{I I c} / \mathcal{G}_{I c}=1$. For instance, the wing cracks initiate at this same flaw corner as $\mathcal{G}_{I I c} / \mathcal{G}_{I c}$ increases. They propagate towards the opening mode-dissipative direction, that is parallel with the zero-traction lateral boundaries. Hence wing crack propagation maximizes fracture dissipation for $\mathcal{G}_{I c}>\mathcal{G}_{I I c}$; peak stress at their initiation, however, is only loosely 


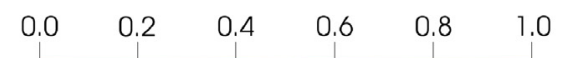

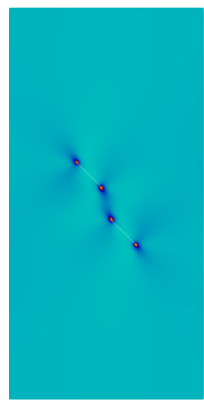
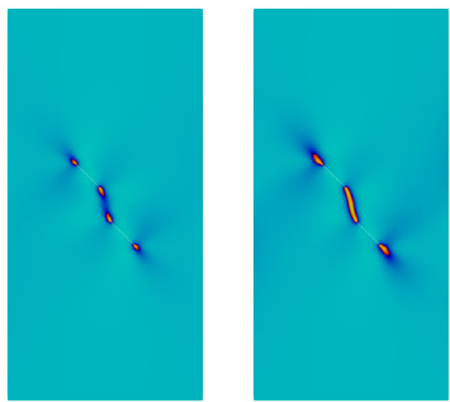

(a) $\mathcal{G}_{I I c} / \mathcal{G}_{I c}=1$, at $u_{2}=200,205,416 \times-10^{-4} \mathrm{~mm}$.
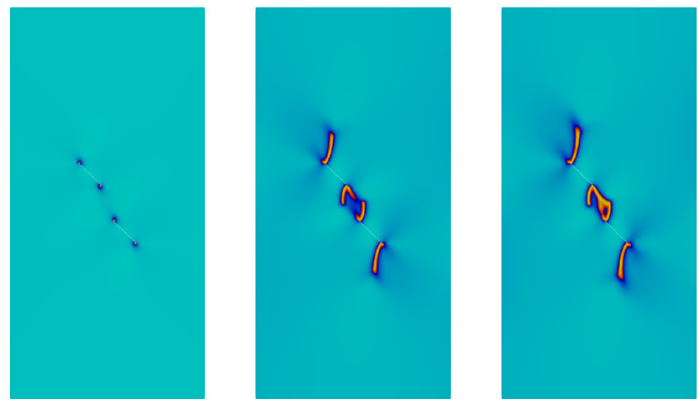

(c) $\mathcal{G}_{I I c} / \mathcal{G}_{I c}=5$, at $u_{2}=260,474,476 \times-10^{-4} \mathrm{~mm}$.
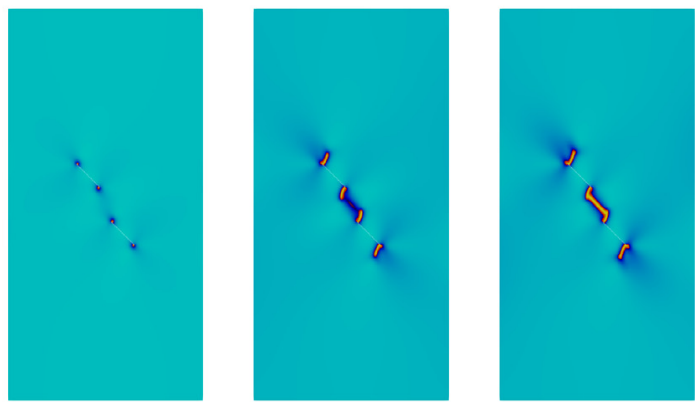

(b) $\mathcal{G}_{I I c} / \mathcal{G}_{I c}=3$, at $u_{2}=255,336,369 \times-10^{-4} \mathrm{~mm}$
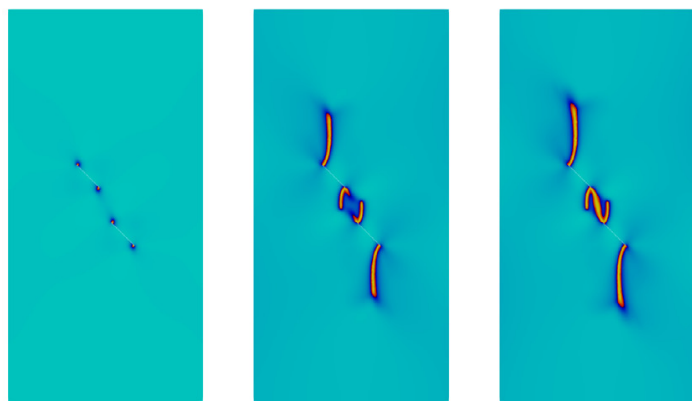

(d) $\mathcal{G}_{I I c} / \mathcal{G}_{I c}=7$, at $u_{2}=265,530,532.5 \times-10^{-4} \mathrm{~mm}$.

Fig. 8. Isotropic mixed-mode case: phase field $d$ for separation $w=6.35 \mathrm{~mm}$, evidencing significant topological change in closure crack geometry between critical fracture energy ratios $\mathcal{G}_{I I c} / \mathcal{G}_{I c}=3$ and 7 .

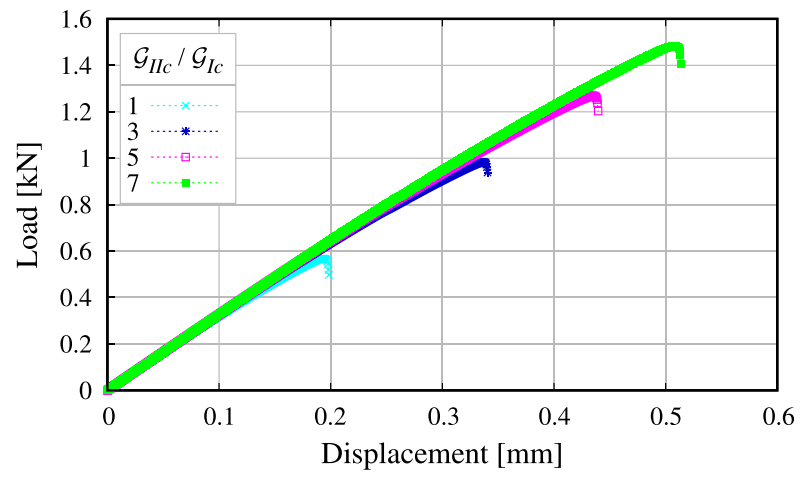

(a) $w=0 \mathrm{~mm}$

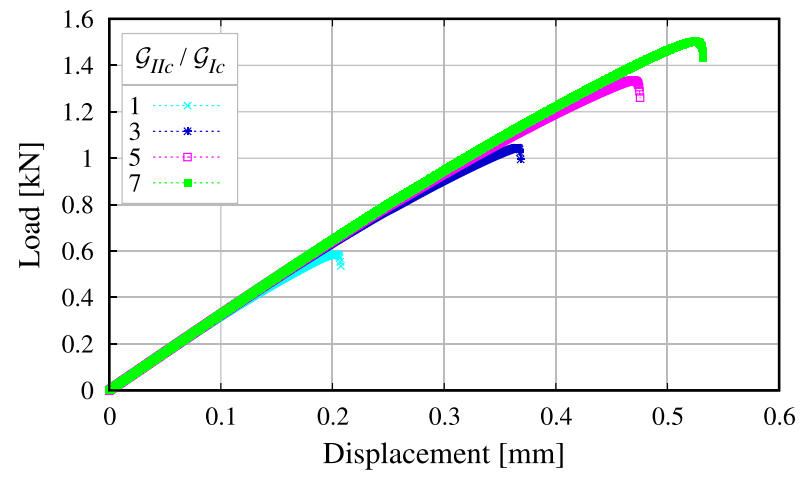

(b) $w=6.35 \mathrm{~mm}$

Fig. 9. Isotropic mixed-mode case: force-displacement curves.

controlled by the baseline value of $\mathcal{G}_{I c}$. After sufficient loading, the stress redistribution due to the wings enhances shear at the original flaw tips. This causes $\mathcal{G}_{I I c}$-sensitive crack bifurcation and hence secondary cracks. Definitively greater mode II fracture energy $\mathcal{G}_{I I c}$ delays secondary crack appearance: compare Fig. 8 cases where $\mathcal{G}_{I I c} / \mathcal{G}_{\text {Ic }}=3$ and 7 , and see $\mathcal{F}$ with increasing $\mathcal{G}_{I I c}$ in Fig. 3. 

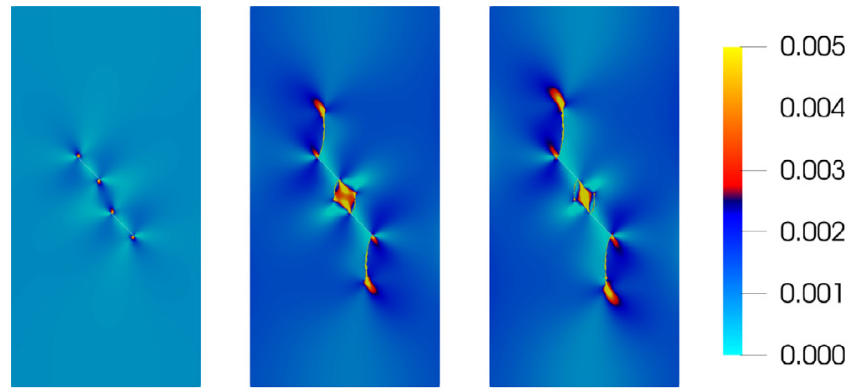

(a) $\mathcal{G}_{I I c} / \mathcal{G}_{I c}=7$, greatest in-plane principal strain.
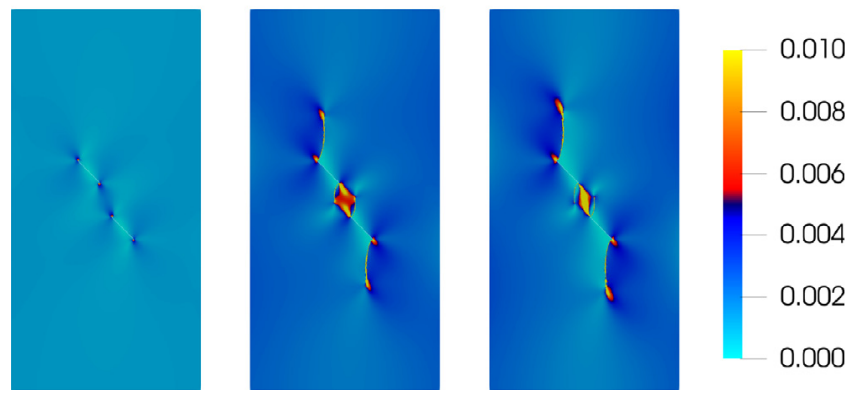

(b) $\mathcal{G}_{I I c} / \mathcal{G}_{I c}=7$, in-plane shear strain.

Fig. 10. Isotropic mixed-mode case: strains for separation $w=6.35 \mathrm{~mm}$, at $u_{2}=265,530,532.5 \times-10^{-4} \mathrm{~mm}$, where in-plane shear strain is the in-plane principal strain difference divided by 2 .
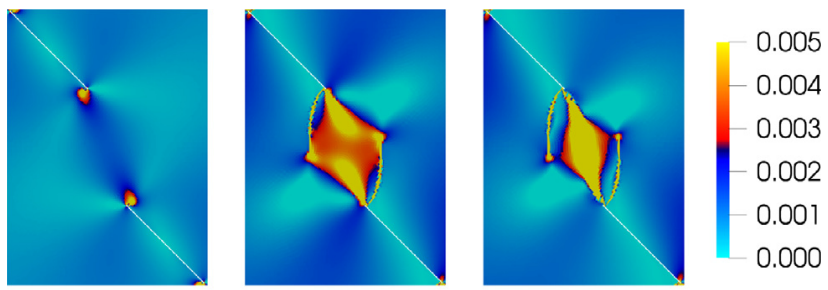

(a) $\mathcal{G}_{I I c} / \mathcal{G}_{I c}=7$, greatest in-plane principal strain
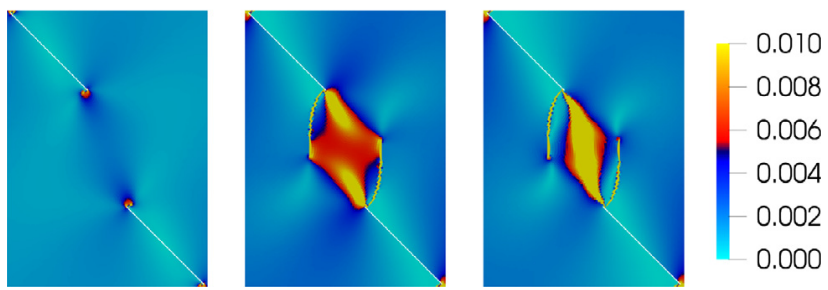

(b) $\mathcal{G}_{I I c} / \mathcal{G}_{I c}=7$, shear in-plane strain.

Fig. 11. Isotropic mixed-mode case: strains for $w=6.35 \mathrm{~mm}$, at $u_{2}=265,530,532.5 \times-10^{-4} \mathrm{~mm}$, with zoom-in region per Fig. 6 .

With a single exception, the fractures' topology is explicable by the principal strains in the near-flaw and nearcrack-tip regions, Figs. 10 and 11. That exception is coalescence for separation $w>0$ at higher ratios $\mathcal{G}_{I I c} / \mathcal{G}_{I c}$, see $\mathcal{G}_{I I c} / \mathcal{G}_{I c}=7$ in Figs. 8 and 10, where coalescent fracture transitions from secondary shear to mixed-mode $x_{1}$-direction opening. Moreover during coalescence, significant material degradation occurs forward of the secondary crack tips, 
with only postcedent mixed-mode brutal crack growth. The wing cracks laterally both bound the forward region and share its opening direction, indicating mode I energy release. By way of addendum, Shen [45] similarly requires recourse to element insertion away from the secondary crack tips, in order to capture coalescence for separation $w>0$ and comparable mixity ratio.

For non-zero flaw separation $w$, therefore, the coalescent fracture geometry is the most sensitive to the parameterization (an observation that shall be echoed in the anisotropic results, as shown in the next section). In contrast for separation $w>0$ and $\mathcal{G}_{I I c} / \mathcal{G}_{I c}=3$, the coalescent fracture develops only in the shear plane, directly between wing crack tips. Alternately for $\mathcal{G}_{I I c} / \mathcal{G}_{I c}=5$ at $u_{2}=476 \times-10^{-4}$, an intermediate result develops: damage in the central coalescent region simultaneous to connecting the wings. The wing cracks are relatively stunted at coalescence, meaning this result is likely attributable to inferior inter-wing stress-shadowing during coalescent fracture. We observe corollary less crack-forward mode I dissipation.

In summary, coalescent fracture contrasts sharply with the baseline no-mixity case. After the wing crack propagation, due to the shearing in-plane $\epsilon$-eigenvalues, $W_{I I}$ resolves as non-zero at the flaw tips. Thus, secondary crack patterns appear with increasing $\mathcal{G}_{I I c}$. Simply put, capturing the consistent kinematics does not only mitigate compactive fracture (see Appendix B), but here also promote secondary bifurcations.

\subsubsection{Type I fracture coalescence pattern}

Overall, the mixed-mode phase field fracture model is capable of capturing coalescence patterns and coalescent propagation sequencing not replicated in some benchmark fracture models, including other energy-based models. Both of the experimentally observed crack coalescence patterns that we investigate are qualitatively recovered: type I for $w=0$, by comparison of Fig. 6(b) to Fig. 7(b); and, type II for $w>0$ in Fig. 8(d). When compared to the experimentally typology, the characteristic features of the type I pattern recovered are: stunted interior wing cracks combined with coalescence in the shear plane between initial flaw tips. However, our simulations also exhibit relatively stunted shear-induced crack propagation at the external flaw tips preceding coalescence, when compared to the counterparts generated from the boundary element method equipped with stress-based crack propagation criterion in [75], as well as to the crack pattern shown in experiments (see Figure 9 of Bobet and Einstein [5]). A strain-based propagation model using an identical discretization and a similar boundary value problem, for which $w=0$ but at a different angle $\gamma$, similarly shows reduced shear-induced damage at the external flaw tips [30]. Meanwhile, simulations using the energy-based displacement discontinuity model (cf. [12,45,13]) either eliminated or reduced external flaw-tip fracture growth for specimens with a variety of pre-existing flaw configurations. In the same vein, the Zhang et al. [14] phase-field model is similarly energy-based and evidences damage at the external flaw tips, but no shear-induced crack propagation from those tips until after coalescence, which is consistent with our numerical simulations.

\subsubsection{Type II fracture coalescence pattern}

In the literature, significant attention has been paid to the recovery of the type II coalescence patterns in numerical simulations. For instance, Reyes and Einstein [8] employs a smeared crack approach after Lemaitre [78] where a damage threshold is set for maximum principal tensile strain such that the damage accumulated with a evolution law once that threshold value is reached. They recover wing cracks, but no secondary-shear cracks are found in their numerical simulations. Shen and co-workers capture the type II pattern with a specimen with flaw angle $\gamma=\pi / 4$. However, their model requires additional evaluations of the modal stress intensity factors in the bridge area between the two initial flaws. They then insert elements disconnected from any existing macroscopically distinct stress singularity (see Figure 3 in [13]). In other words, in these previous simulations, the coalescent crack propagates into the secondary shear cracks, and not visa versa, as the crack nucleates in opening mode I. On the other hand, in order to obtain the characteristic anti-symmetric type II closure geometry of Fig. 6(b), the strain- and stress-criterion propagation mechanism-based simulations reverse that sequence. The secondary shear cracks coalesce towards each other, after abruptly re-orienting into the opening mode, rather than outward from the centroid of the domain, compare with Figure 14 in [75]. Both phase-field models also appear to propagate towards the opening mode, although some inter-flaw softening in the bridge area is acknowledged, also see Fig. 11(a). Thus, a qualitative comparison - the type of which has previously been performed for strain- versus stress-based propagation models in [30] - reveals differences in the sequencing of the evolved coalescent cracks, depending on the simulation model employed. As a caveat, from the existing literature and even when provided high-quality images, it is difficult to disambiguate precedence: cf. the partial photographic time series of coalescence in gypsum, introduced in Fig. 1(a-c) [4]. 


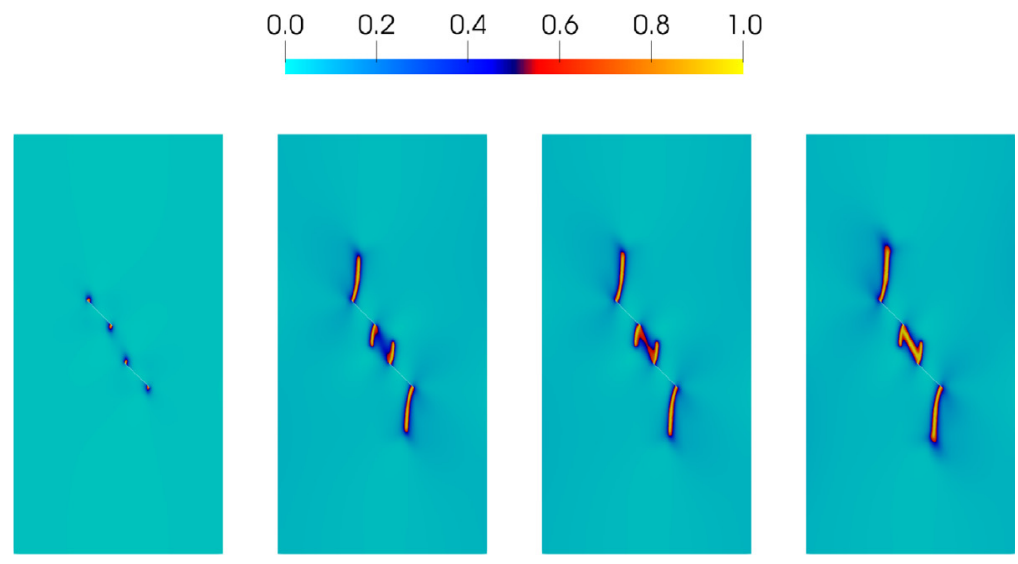

(a) $\theta=0$, at $u_{2}=230,420,423,425 \times-10^{-4} \mathrm{~mm}$.
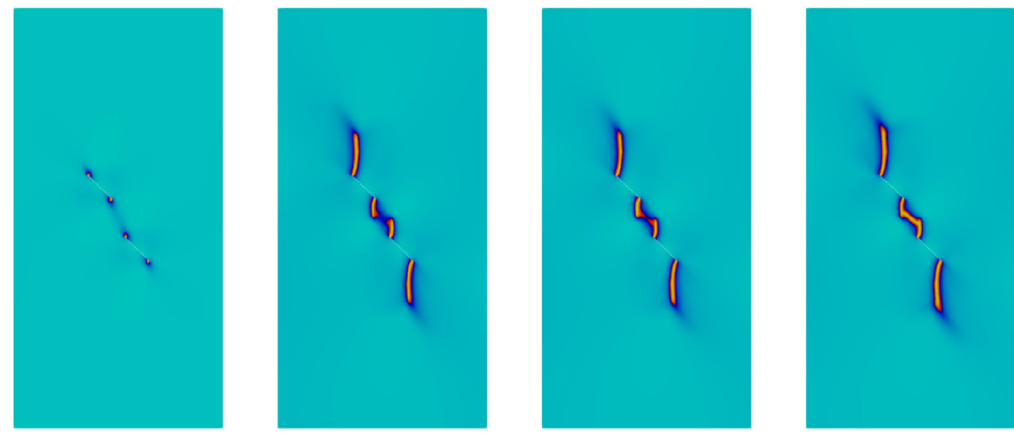

(b) $\theta=\pi / 4$, at $u_{2}=289,444,445,447 \times-10^{-4} \mathrm{~mm}$.
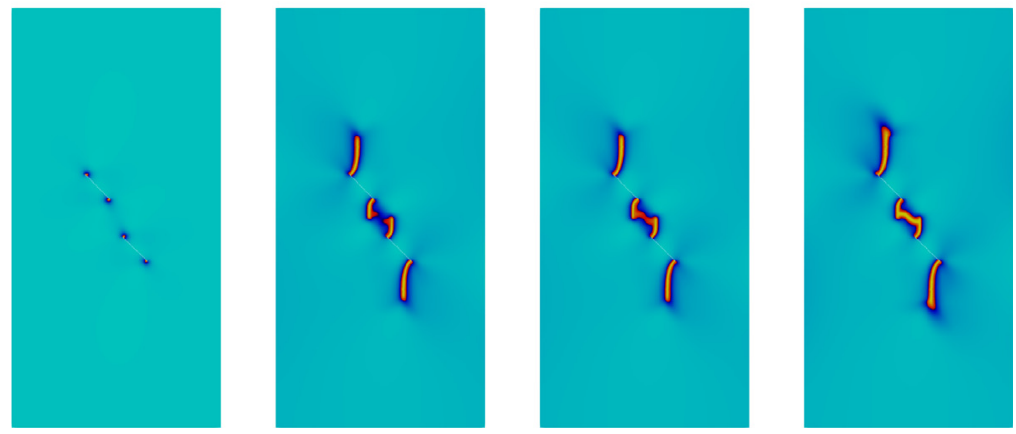

(c) $\theta=\pi / 2$, at $u_{2}=290,545,546,548 \times-10^{-4} \mathrm{~mm}$.

Fig. 12. Anisotropic mixed-mode case:phase field $d$ for separation $w=6.35 \mathrm{~mm}$, critical fracture energy ratio $\mathcal{G}_{I I c} / \mathcal{G}_{I c}=7$, and phase-field anisotropic coefficient $\beta=1$.

\subsection{Anisotropic coalescent case}

Anisotropic parameters have essentially been chosen to exhibit smooth, controllable deviation from the isotropic mixed-mode coalescent case, above. The anisotropic coalescent cases are that case with the following modifications: $\chi=\lambda$, fracture energy diffusion parameter $\beta=1$, and fixed $\mathcal{G}_{I I c} / \mathcal{G}_{I c}=7$ at the mode I-coalescent threshold. The parameterization is the microstructural angle $\theta_{l}$, which increases from 0 to $\pi / 2$. 


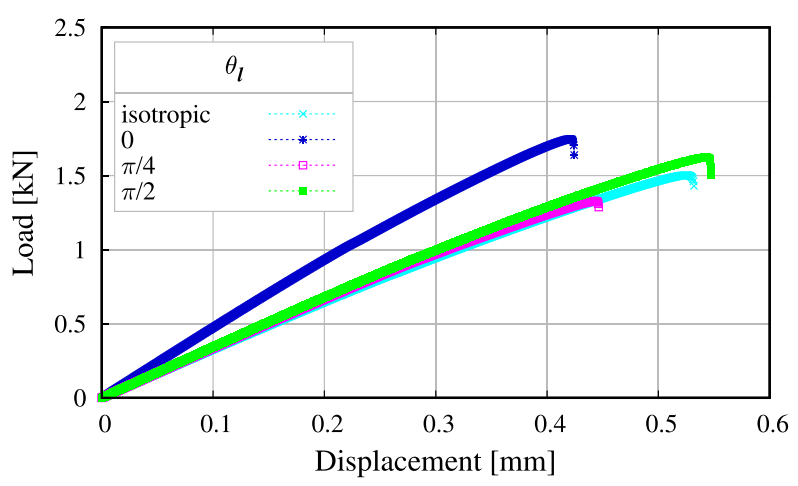

Fig. 13. Anisotropic mixed-mode case: force-displacement curves.

Mode-mixity significantly impacts fracture growth, particularly in the near-flaw region for these cases. Fracture initiates in the same locations as previous, as shown in Fig. 12. Once again, the wing cracks develop first. Anisotropy significantly alters the wing cracks' propagation, however. For case $\theta_{l}=0$ with highest in-plane stiffness in the $x_{2}-$ direction, the inter-flaw wings exhibit near-direct reorientation towards $x_{2}$-direction growth. However the coalescent pattern almost repeats the isotropic case, if at an enhanced peak stress, as shown in Fig. 13.

For the case $\theta_{l}=\pi / 4$ in contrast, the fractures coalesce in the shear plane, between the wing cracks' tips. Similarly for case $\theta_{l}=\pi / 2$, we also observe severely stunted inter-closure wing cracks. Hence a rotated structural direction masks the complex inter-wing coalescent behavior, otherwise observable under isotropy at the same $\mathcal{G}_{I I c} / \mathcal{G}_{I c}$. Instead, smaller wing cracks lead to shear-plane coalesce between the wings' tips.

\section{Conclusion}

We present a phase field fracture framework to replicate secondary cracks by introducing distinctive critical energy release rates for different kinematic modes in the brittle regime. To the best of the authors' knowledge, this is the first phase field fracture model that captures these cracks using a consistent kinematic argument. This is significant not only for theoretical consistency, but also for inferring numerical values of the critical energy release rates measured from specimens subjected to mode I and mode II loadings. We also provide a theoretical basis for the governing equation of phase field via a microforce balance. This formulation allows us to obtain the driving force from a local dissipation maximization problem where the crack propagation direction is determined. Hence, the kinematics mode for the crack is consistent. This mathematical framework is applied to both isotropic and transverse-isotropic materials. Numerical examples demonstrate that (1) a transition from shear-coalescent to mixed-coalescent cracks may occur by varying the ratio of the critical energy release rate for different modes and (2) the formation of wing cracks and secondary cracks with the consistent kinematic modes can be captured for both isotropic and transversely isotropic materials.

\section{Acknowledgments}

The authors thank Professor John Williams from MIT for the fruitful discussion that motivate us to write this paper. This research is supported by the Earth Materials and Processes program from the US Army Research Office under grant contract W911NF-15-1-0442 and W911NF-15-1-0581, the Dynamic Materials and Interactions program from the Air Force Office of Scientific Research under grant contract FA9550-17-1-0169, the Nuclear Energy University program from the Department of Energy under grant contract DE-NE0008534 as well as the Mechanics of Materials and Structures program at National Science Foundation under grant contract CMMI-1462760. These supports are gratefully acknowledged. The views and conclusions contained in this document are those of the authors, and should not be interpreted as representing the official policies, either expressed or implied, of the sponsors, including the Army Research Laboratory or the U.S. Government. The U.S. Government is authorized to reproduce and distribute reprints for Government purposes notwithstanding any copyright notation herein. 


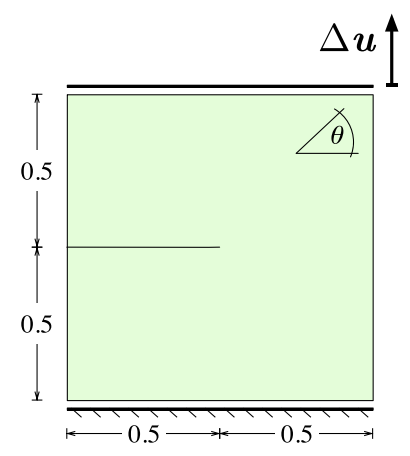

Fig. 14. Setup of the boundary value problem used in both the spurious healing and shearing fracture simulations.

\section{Appendix A. Spurious crack healing case}

Our simplest numerical example compares time series of crack-healing results versus not. This example addresses the idea to store the maximum value of $\mathcal{H}$ over the time history, to putatively stop crack healing. For $\mathcal{G}_{I c} \neq \mathcal{G}_{I I c}$, crack healing can be demonstrated numerically. In particular for $\mathcal{G}_{I c} \ll \mathcal{G}_{I I c}$, we select a problem similar to the commonly used pure shear benchmark problem in [29]: albeit with mixed-mode boundary conditions, Fig. 14.

The elastic material parameters are: $\lambda=121.15 \mathrm{kN} / \mathrm{mm}^{2}$ and $\mu=80.77 \mathrm{kN} / \mathrm{mm}^{2}$. The numerical parameters are $l=15 \times 10^{-3} \mathrm{~mm}$ for a mesh universally refined to $3.90625 \times 10^{-3} \mathrm{~mm}$. Elastic and numerical parameters the same for all cases using loading Fig. 14, unless otherwise noted. The mode I critical energy release rate is $\mathcal{G}_{I c}=2.7 \times 10^{-3}$ $\mathrm{kN} / \mathrm{mm}$, whereas $\mathcal{G}_{I I c}=2.7 \times 10^{-1} \mathrm{kN} / \mathrm{mm}$. Note the lateral left and right boundaries are traction free. On the bottom boundary $\Delta u_{1}=\Delta u_{2}=0 \mathrm{~mm}$ for all time steps. On the top boundary we prescribe: $\Delta u_{1}=0 \mathrm{~mm}$ and $\Delta u_{2}=1.0 \times 10^{-5}$ for the first 940 time steps; $\Delta u_{1}=0 \mathrm{~mm}$ and $\Delta u_{2}=-1.0 \times 10^{-5} \mathrm{~mm}$ for the next 940 time steps; and, $\Delta u_{1}=1.0 \times 10^{-5} \mathrm{~mm}$ and $\Delta u_{2}=0$ for the final 3120 time steps. The effect of crack healing can be seen by comparison of Fig. 15. The spurious healing is visible, and can be discerned from local reductions in the damage variable towards the tensile fracture tip.

If the history function is not as assumed in Eq. (21), then spurious crack healing may occur when $\mathcal{G}_{\text {Ic }} \neq \mathcal{G}_{\text {IIc }}$. For the case where $\mathcal{G}_{I c} \ll \mathcal{G}_{I I c}$ as above, the spurious healing may occur if the external force is not monotonically increasing. In this numerical example the external force follows the following sequence: primary tensile loading, unloading, and lastly re-loading in primary shear. The numerical-experimental premise is to drive up $\mathcal{H}_{I}$ for a relatively low value of $\mathcal{G}_{I c}$ vs. $\mathcal{G}_{I I c}$. Subsequently we unload, so that current values of $\mathcal{H}$ exceed the corresponding stored energy partition due to a legacy of tensile loading. Now loading in pure shear, at some point we increase the current $\mathcal{H}$ (now due to increases in $\mathcal{H}_{I I}$ ) beyond the historical value (due to legacy $\mathcal{H}_{I}$ ). But as $\mathcal{G}_{I I c} \gg \mathcal{G}_{I c}$ for this example, the critical-energy-normalized combination of $\mathcal{H}_{I}$ and $\mathcal{H}_{I I}$ plunges. So does the crack heal.

\section{Appendix B. Shear case}

In this work, we decompose the strain energy functional in a new manner. Certainly we do not follow prior ideas e.g. [73], except in its broadest outlines. Therefore, the no-mixity $\mathcal{G}_{I c}=\mathcal{G}_{I I c}$ result cannot be anticipated to exactly recover Miehe et al. [73]-type behavior. Overall, when the body is loaded quasi-statically in mixed-mode loading, we desire that the induced fracture not branch. This well-known problem with the phase field fracture approximation traces to Bourdin et al. [15]. Compressive-zone fracture can alternately be mitigated by recourse to a volumetric/deviatoric split Amor et al. [79].

This boundary-value problem addresses inhibition of fracture growth in compressive zones. Specifically we prescribe $\Delta u_{1}=1.0 \times 10^{-5} \mathrm{~mm}$ and $\Delta u_{2}=0$ for all 1500 time steps. A comparison of the results is presented in Fig. 16. They evidence a single crack tip emerging from the initial discontinuity, unlike prior so-called "isotropic" model like Bourdin et al. [15]. In short, our strain decomposition also eliminates undesirable crack initiation in compressive zones, Fig. 17. 


$\begin{array}{llllll}0.0 & 0.2 & 0.4 & 0.6 & 0.8 & 1.0\end{array}$
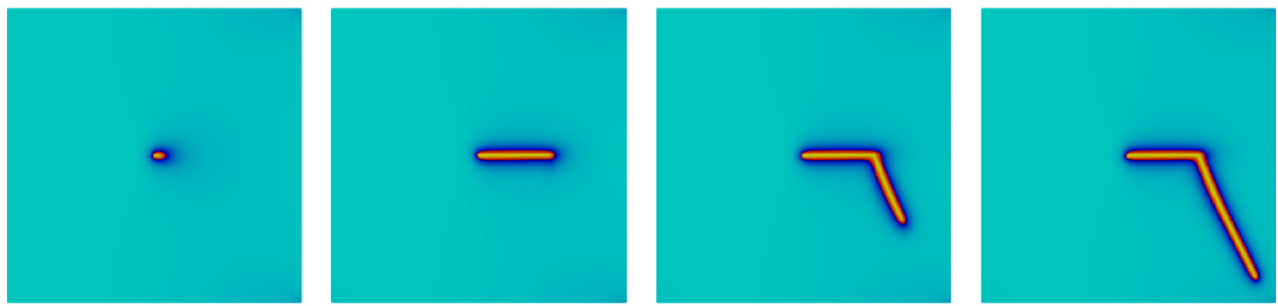

(a) Irreversible model using combined history function of Eq. (21), left-to-right: initial tensile loading, tensile unloading, subsequent shearing, and final loading step.
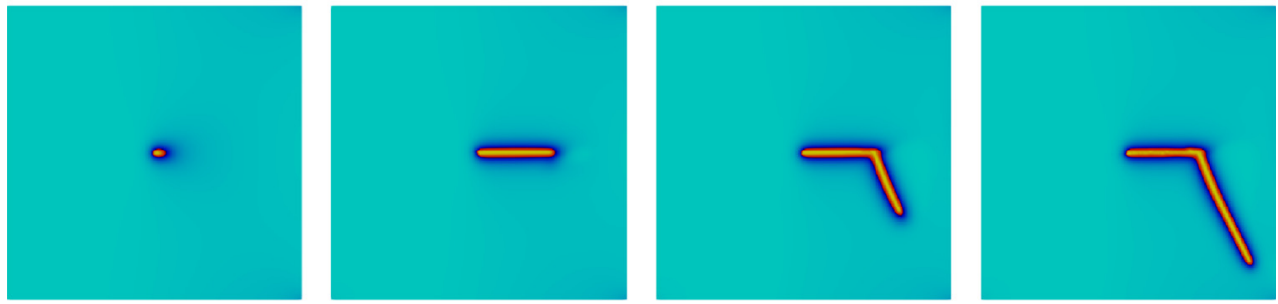

(b) Healing model using dual history functions of Eqs. (1) and (2), left-to-right: same loadings as the above.
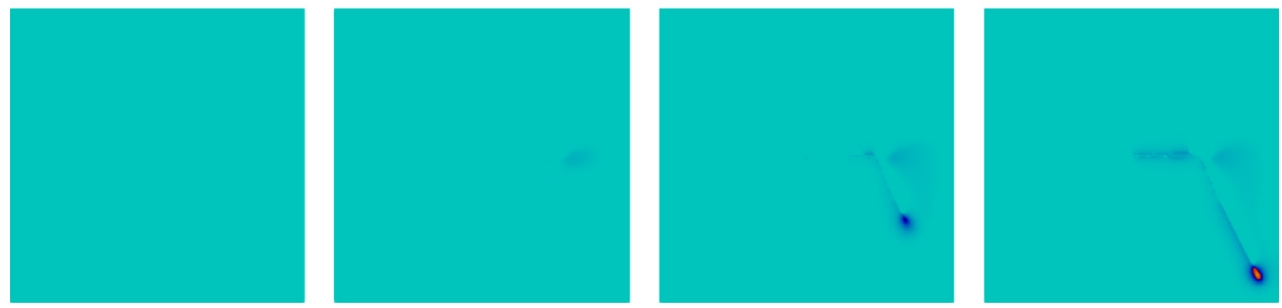

(c) Difference of (a) minus (b)'s phase field.

Fig. 15. Consistency case phase field $d$, at $u_{1}=900 \times 10^{-5}$ and $u_{2}=1620,1820,2620 \times 10^{-5} \mathrm{~mm}$.

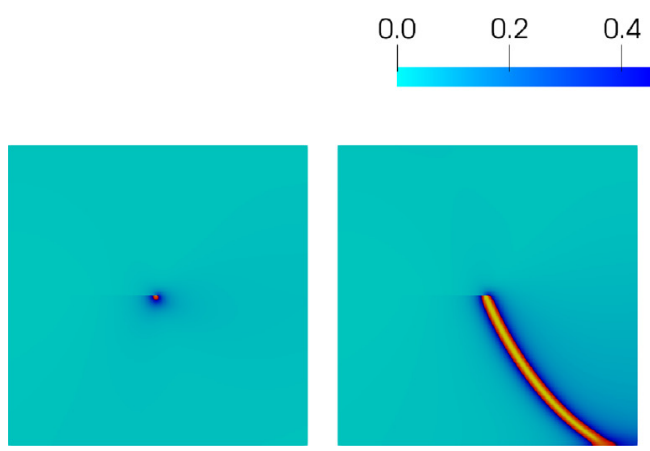

(a) $\mathcal{G}_{I I c} / \mathcal{G}_{I c}=1$.
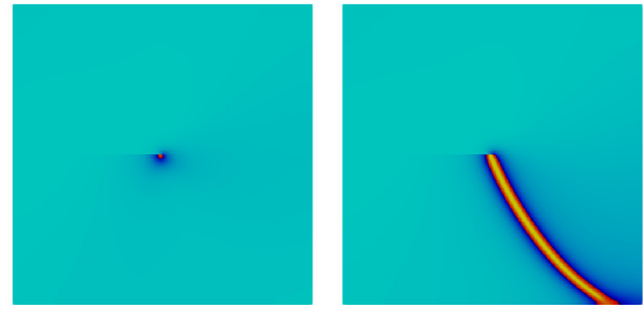

(b) $\mathcal{G}_{I I c} / \mathcal{G}_{I c}=7$.

Fig. 16. Shear case phase field $d$, at $u_{2}=850,1340 \times 10^{-5} \mathrm{~mm}$. 


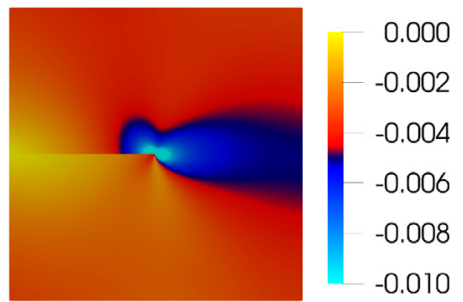

Fig. 17. Shear case for critical fracture energy ratio $\mathcal{G}_{I I c} / \mathcal{G}_{I c}=7$, least in-plane principal strain, at $u_{2}=850 \times 10^{-5} \mathrm{~mm}$.

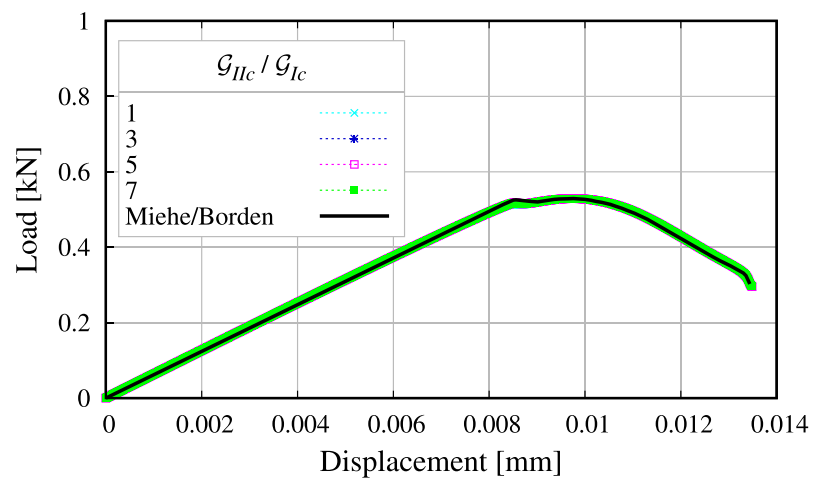

Fig. 18. Shear case force-displacement curves, overlapping for all critical fracture energy ratios $\mathcal{G}_{I I c} / \mathcal{G}_{I c}$, with comparison to the original Miehe et al. [29] taking $\mathcal{G}_{c}=\mathcal{G}_{I c}$ and regular vs. isogeometric finite element analysis [31], elsewhere used for e.g. numerical benchmarking of mesh h-adaptivity [80].

However, another significant trend is noticeable. The crack propagates in a zone without resolving a maximizing shear strain. In return, the fracture trajectories as well as force-displacement curves are basically unaffected by the ratio $\mathcal{G}_{I I c} / \mathcal{G}_{I c}$, Fig. 18. The local dissipation maximization-based routine both qualitatively as well as quantitatively recovers the result of strain eigenvalue-based partition [29]; this problem has been previously studied using both that and the volumetric/deviatoric partitions, cf. [31,49,80,81], among others.

That combination is both sensible and desirable. Inasmuch as the material stiffness degrades in a region due to opening, the preponderance of near-tip energy dissipation is due to mode I fracture (as here). Moreover since we ascribe kinematic consistency to this isotropic material, $\theta$ maximizing $\mathcal{F}$ resolves zero shear. So $\mathcal{F}=W_{I} / \mathcal{G}_{I c}+0 / \mathcal{G}_{I I c}$ at the fracture tip, refer to Fig. 3. Hence increasing $\mathcal{G}_{I I c}$ causes only slight changes in this result, as demonstrated in the overlapping force-displacement curves for all ratios $\mathcal{G}_{I I c} / \mathcal{G}_{I c}$. This outcome is as per our expectation, yet very dichotomous from fracturing in a compactive region (as with coalescent cases, Section 3).

\section{References}

[1] A.A. Griffith, The phenomena of rupture and flow in solids, Phil. Trans. R. Soc. A 221 (582-593) (1921) 163-198.

[2] J.W. Rudnicki, Fracture mechanics applied to the Earth's crust, Annu. Rev. Earth Planet. Sci. 8 (1) (1980) 489-525.

[3] J.W. Hutchinson, Z. Suo, Mixed mode cracking in layered materials, Adv. Appl. Mech. 29 (1991) 63-191.

[4] L.D. Suits, T.C. Sheahan, L.N.Y. Wong, H.H. Einstein, Using high speed video imaging in the study of cracking processes in rock, Geotech. Test. J. 32 (2) (2009) 164-180.

[5] A. Bobet, H.H. Einstein, Fracture coalescence in rock-type materials under uniaxial and biaxial compression, Int. J. Rock Mech. Min. Sci. 35 (7) (1998) 863-888.

[6] A. Bobet, Numerical simulation of initiation of tensile and shear cracks, in: DC Rocks 2001, the 38th US Symposium on Rock Mechanics (USRMS), American Rock Mechanics Association, 2001.

[7] S.Q. Yang, D.S. Yang, H.W. Jing, Y.H. Li, S.Y. Wang, An experimental study of the fracture coalescence behaviour of brittle sandstone specimens containing three fissures, Rock Mech. Rock Eng. 45 (4) (2012) 563-582.

[8] O. Reyes, H.H. Einstein, Failure mechanisms of fractured rock - a fracture coalescence model, in: 7th International Conference on Rock Mechanics, International Society for Rock Mechanics, 1991, pp. 333-340. 
[9] B. Vásárhelyi, A. Bobet, Modeling of crack initiation, propagation and coalescence in uniaxial compression, Rock Mech. Rock Eng. 33 (2) (2000) 119-139.

[10] H. Liebowitz, G.C. Sih, Mathematical Theories of Brittle Fracture, Academic Press, 1968.

[11] R.J. Nuismer, An energy release rate criterion for mixed mode fracture, Int. J. Fract. 11 (2) (1975) 245-250.

[12] B. Shen, O. Stephansson, Modification of the G-criterion for crack propagation subjected to compression, Eng. Fract. Mech. 47 (2) (1994) 177-189.

[13] B. Shen, O. Stephansson, H.H. Einstein, B. Ghahreman, Coalescence of fractures under shear stresses in experiments, J. Geophys. Res. Solid Earth 100 (B4) (1995) 5975-5990.

[14] X. Zhang, S.W. Sloan, C. Vignes, D. Sheng, A modification of the phase-field model for mixed mode crack propagation in rock-like materials, Comput. Methods Appl. Mech. Engrg. 322 (2017) 123-136.

[15] B. Bourdin, G.A. Francfort, J.J. Marigo, The variational approach to fracture, J. Elasticity 91 (1-3) (2008) 5-148.

[16] C. Linder, F. Armero, Finite elements with embedded branching, Finite Elem. Anal. Des. 45 (4) (2009) $280-293$.

[17] A.R. Khoei, Extended Finite Element Method: Theory and Applications, John Wiley \& Sons, 2014.

[18] N. Moës, C. Stolz, P.E. Bernard, N. Chevaugeon, A level set based model for damage growth: The thick level set approach, Internat. J. Numer. Methods Engrg. 86 (3) (2011) 358-380.

[19] A. Pandolfi, M. Ortiz, An eigenerosion approach to brittle fracture, Internat. J. Numer. Methods Engrg. 92 (8) (2012) 694-714.

[20] T. Belytschko, W.K. Liu, B. Moran, K. Elkhodary, Nonlinear Finite Elements for Continua and Structures, John Wiley \& Sons, 2013.

[21] W.C. Sun, Z. Cai, J. Choo, Mixed Arlequin method for multiscale poromechanics problems, Internat. J. Numer. Methods Engrg. 111 (2017) $624-659$.

[22] G.C. Sih, Some basic problems in fracture mechanics and new concepts, Eng. Fract. Mech. 5 (2) (1973) 365-377.

[23] C. Callari, F. Armero, Finite element methods for the analysis of strong discontinuities in coupled poro-plastic media, Comput. Methods Appl. Mech. Engrg. 191 (39) (2002) 4371-4400.

[24] R.I. Borja, Finite element simulation of strain localization with large deformation: capturing strong discontinuity using a Petrov-Galerkin multiscale formulation, Comput. Methods Appl. Mech. Engrg. 191 (27) (2002) 2949-2978.

[25] R.I. Borja, Assumed enhanced strain and the extended finite element methods: A unification of concepts, Comput. Methods Appl. Mech. Engrg. 197 (33) (2008) 2789-2803.

[26] N. Sukumar, N. Moës, B. Moran, T. Belytschko, Extended finite element method for three-dimensional crack modelling, Internat. J. Numer. Methods Engrg. 48 (11) (2000) 1549-1570.

[27] A. Pandolfi, P.R. Guduru, M. Ortiz, A.J. Rosakis, Three dimensional cohesive-element analysis and experiments of dynamic fracture in C300 steel, Int. J. Solids Struct. 37 (27) (2000) 3733-3760.

[28] R.I. Borja, Plasticity: Modeling \& Computation, Springer Science \& Business Media, 2013.

[29] C. Miehe, M. Hofacker, F. Welschinger, A phase field model for rate-independent crack propagation: Robust algorithmic implementation based on operator splits, Comput. Methods Appl. Mech. Engrg. 199 (45-48) (2010) 2765-2778.

[30] B.G. da Silva, H.H. Einstein, Modeling of crack initiation, propagation and coalescence in rocks, Int. J. Fract. 182 (2) (2013) $167-186$.

[31] J.B. Michael, C.V. Verhoosel, M.A. Scott, T.J.R. Hughes, C.M. Landis, A phase-field description of dynamic brittle fracture, Comput. Methods Appl. Mech. Engrg. 217 (2012) 77-95.

[32] T. Heister, M.F. Wheeler, T. Wick, A primal-dual active set method and predictor-corrector mesh adaptivity for computing fracture propagation using a phase-field approach, Comput. Methods Appl. Mech. Engrg. 290 (2015) 466-495.

[33] S. Teichtmeister, D. Kienle, F. Aldakheel, M.A. Keip, Phase field modeling of fracture in anisotropic brittle solids, Int. J. Non-Linear Mech. 97 (2017) 1-21.

[34] L. Ambrosio, V.M. Tortorelli, Approximation of functional depending on jumps by elliptic functional via t-convergence, Comm. Pure Appl. Math. 43 (8) (1990) 999-1036.

[35] S. Lee, M.F. Wheeler, T. Wick, Pressure and fluid-driven fracture propagation in porous media using an adaptive finite element phase field model, Comput. Methods Appl. Mech. Engrg. 305 (2016) 111-132.

[36] M.J. Borden, T.J.R. Hughes, C.M. Landis, C.V. Verhoosel, A higher-order phase-field model for brittle fracture: formulation and analysis within the isogeometric analysis framework, Comput. Methods Appl. Mech. Engrg. 273 (2014) 100-118.

[37] I. Khisamitov, G. Meschke, Variational approach to interface element modeling of brittle fracture propagation, Comput. Methods Appl. Mech. Engrg. 328 (2018) 452-476.

[38] S. Na, W.C. Sun, Computational thermomechanics of crystalline rock, part I: a combined multi-phase-field/crystal plasticity approach for single crystal simulations, Comput. Methods Appl. Mech. Engrg. 338 (2018) 657-691.

[39] O. Gültekin, H. Dal, G.A. Holzapfel, A phase-field approach to model fracture of arterial walls: theory and finite element analysis, Comput. Methods Appl. Mech. Engrg. 312 (2016) 542-566.

[40] J.D. Clayton, J. Knap, A geometrically nonlinear phase field theory of brittle fracture, Int. J. Fract. 189 (2) (2014) 139-148.

[41] J.D. Clayton, J. Knap, Phase field modeling of directional fracture in anisotropic polycrystals, Comput. Mater. Sci. 98 (2015) 158-169.

[42] J.D. Clayton, J. Knap, Phase field modeling and simulation of coupled fracture and twinning in single crystals and polycrystals, Comput. Methods Appl. Mech. Engrg. 312 (2016) 447-467.

[43] J. Choo, W.C. Sun, Coupled phase-field and plasticity modeling of geological materials: from brittle fracture to ductile flow, Comput. Methods Appl. Mech. Engrg. 330 (2018) 1-32.

[44] J. Choo, W.C. Sun, Cracking and damage from crystallization in pores: coupled chemo-hydro-mechanics and phase-field modeling, Comput. Methods Appl. Mech. Engrg. 335 (2018) 347-379.

[45] B. Shen, The mechanism of fracture coalescence in compression-experimental study and numerical simulation, Eng. Fract. Mech. 51 (1) (1995) 73-85. 
[46] Z.A. Wilson, M.J. Borden, C.M. Landis, A phase-field model for fracture in piezoelectric ceramics, Int. J. Fract. 183 (2) (2013) $135-153$.

[47] Z.A. Wilson, C.M. Landis, Phase-field modeling of hydraulic fracture, J. Mech. Phys. Solids 96 (2016) 264-290.

[48] C. Kuhn, R. Müller, A continuum phase field model for fracture, Eng. Fract. Mech. 77 (18) (2010) 3625-3634.

[49] M. Ambati, T. Gerasimov, L. De Lorenzis, A review on phase-field models of brittle fracture and a new fast hybrid formulation, Comput. Mech. 55 (2) (2014) 383-405.

[50] K. Wang, W.C. Sun, A unified variational eigen-erosion framework for interacting brittle fractures and compaction bands in fluid-infiltrating porous media, Comput. Methods Appl. Mech. Engrg. 318 (2017) 1-32.

[51] M.E. Gurtin, Generalized Ginzburg-Landau and Cahn-Hilliard equations based on a microforce balance, Physica D 92 (3-4) (1996) 178-192.

[52] O. Gültekin, H. Dal, G.A. Holzapfel, Numerical aspects of anisotropic failure in soft biological tissues favor energy-based criteria: A ratedependent anisotropic crack phase-field model, Comput. Methods Appl. Mech. Engrg. 331 (2018) 23-52.

[53] C. Miehe, S. Mauthe, S. Teichtmeister, Minimization principles for the coupled problem of Darcy-Biot-type fluid transport in porous media linked to phase field modeling of fracture, J. Mech. Phys. Solids 82 (2015) 186-217.

[54] C. Miehe, L.M. Schänzel, H. Ulmer, Phase field modeling of fracture in multi-physics problems. Part I. Balance of crack surface and failure criteria for brittle crack propagation in thermo-elastic solids, Comput. Methods Appl. Mech. Engrg. 294 (2015) 449-485.

[55] T. Backers, O. Stephansson, ISRM suggested method for the determination of mode II fracture toughness, Rock Mech. Rock Eng. 45 (6) (2012) 137-163.

[56] J.Y. Wu, M. Cervera, On the equivalence between traction- and stress-based approaches for the modeling of localized failure in solids, J. Mech. Phys. Solids 82 (2015) 137-163.

[57] H. Niandou, J.F. Shao, J.P. Henry, D. Fourmaintraux, Laboratory investigation of the mechanical behaviour of Tournemire shale, Int. J. Rock Mech. Min. Sci. 34 (1) (1997) 3-16.

[58] S.J. Semnani, J.A. White, R.I. Borja, Thermoplasticity and strain localization in transversely isotropic materials based on anisotropic critical state plasticity, Int. J. Numer. Anal. Methods Geomech. 40 (18) (2016) 2423-2449.

[59] S. Na, W.C. Sun, M.D. Ingraham, H. Yoon, Effects of spatial heterogeneity and material anisotropy on the fracture pattern and macroscopic effective toughness of Mancos shale in Brazilian tests, J. Geophys. Res. Solid Earth 122 (8) (2017) 6202-6230.

[60] L.J. Walpole, Fourth-rank tensors of the thirty-two crystal classes: multiplication tables, Proc. R. Soc. A Math. Phys. Eng. Sci. 391 (1800) (1984) 149-179.

[61] B. Schmidt, F. Fraternali, M. Ortiz, Eigenfracture: an eigendeformation approach to variational fracture, Multiscale Model. Simul. 7 (3) (2009) $1237-1266$.

[62] A. Pandolfi, B. Li, M. Ortiz, Modeling fracture by material-point erosion, Int. J. Fract. 184 (1-2) (2013) 3-16.

[63] Y. Liu, V. Filonova, N. Hu, Z. Yuan, J. Fish, Z. Yuan, T. Belytschko, A regularized phenomenological multiscale damage model, Internat. J. Numer. Methods Engrg. 99 (12) (2014) 867-887.

[64] R. Radovitzky, A. Seagraves, M. Tupek, L. Noels, A scalable 3D fracture and fragmentation algorithm based on a hybrid, discontinuous Galerkin, cohesive element method, Comput. Methods Appl. Mech. Engrg. 200 (1-4) (2011) 326-344.

[65] B. Shen, O. Stephansson, M. Rinne, Modelling Rock Fracturing Processes, Springer Netherlands, Dordrecht, 2014 , p. 181.

[66] J.N. Lyness, C.B. Moler, Numerical differentiation of analytic functions, SIAM J. Numer. Anal. 4 (2) (1967) $202-210$.

[67] M. Tanaka, M. Fujikawa, D. Balzani, J. Schröder, Robust numerical calculation of tangent moduli at finite strains based on complex-step derivative approximation and its application to localization analysis, Comput. Methods Appl. Mech. Engrg. 269 (2014) 454-470.

[68] M.D. Brothers, J.T. Foster, H.R. Millwater, A comparison of different methods for calculating tangent-stiffness matrices in a massively parallel computational peridynamics code, Comput. Methods Appl. Mech. Engrg. 279 (2014) 247-267.

[69] W. Bangerth, R. Hartmann, G. Kanschat, deal.II -a general purpose object-oriented finite element library, ACM Trans. Math. Software 33 (4) (2007) 24/1-24/27.

[70] J.A. White, R.I. Borja, Stabilized low-order finite elements for coupled solid-deformation/fluid-diffusion and their application to fault zone transients, Comput. Methods Appl. Mech. Engrg. 197 (49-50) (2008) 4353-4366.

[71] J. Choo, J.A. White, R.I. Borja, Hydromechanical modeling of unsaturated flow in double porosity media, Int. J. Geomech. (2016) D4016002.

[72] S. Na, W.C. Sun, Computational thermo-hydro-mechanics for multiphase freezing and thawing porous media in the finite deformation range, Comput. Methods Appl. Mech. Engrg. 318 (2017) 667-700.

[73] C. Miehe, F. Welschinger, M. Hofacker, Thermodynamically consistent phase-field models of fracture: variational principles and multi-field FE implementations, Internat. J. Numer. Methods Engrg. 83 (10) (2010) 1273-1311.

[74] M.F. Wheeler, T. Wick, W. Wollner, An augmented-Lagrangian method for the phase-field approach for pressurized fractures, Comput. Methods Appl. Mech. Engrg. 271 (2014) 69-85.

[75] A. Bobet, H.H. Einstein, Numerical modeling of fracture coalescence in a model rock material, Int. J. Fract. 92 (3) (1998) $221-252$.

[76] J.M. Sargado, E. Keilegavlen, I. Berre, J.M. Nordbotten, High-accuracy phase-field models for brittle fracture based on a new family of degradation functions, J. Mech. Phys. Solids 111 (2018) 458-489.

[77] M. Negri, C. Ortner, Quasi-static crack propagation by Griffith's criterion, Math. Models Methods Appl. Sci. 18 (11) (2008) $1895-1925$.

[78] Jean Lemaitre, Local approach of fracture, Eng. Fract. Mech. 25 (5-6) (1986) 523-537.

[79] H. Amor, J.J. Marigo, C. Maurini, Regularized formulation of the variational brittle fracture with unilateral contact: numerical experiments, J. Mech. Phys. Solids 57 (8) (2009) 1209-1229.

[80] T. Heister, M.F. Wheeler, T. Wick, Stabilized low-order finite elements for coupled solid-deformation/fluid-diffusion and their application to fault zone transients, Comput. Methods Appl. Mech. Engrg. 290 (2015) 466-495.

[81] S. May, J. Vignollet, R. de Borst, A numerical assessment of phase-field models for brittle and cohesive fracture: $\Gamma$-convergence and stress oscillations, Eur. J. Mech. A. Solids 52 (2015) 72-84. 